\title{
Intramuscular injection of vectorized- scFvMC1 reduces pathological tau in two different tau transgenic models
}

\author{
Francesca Vitale', Jasmin Ortolan', Bruce T. Volpe ${ }^{2,3}$, Philippe Marambaud ${ }^{1,2}$, Luca Giliberto ${ }^{1,2,4^{*}}$ and \\ Cristina d'Abramo ${ }^{1,2^{*}}$ (iD
}

\begin{abstract}
With evidence supporting the prion-like spreading of extracellular tau as a mechanism for the initiation and progression of Alzheimer's disease (AD), immunotherapy has emerged as a potential disease-modifying strategy to target tau. Many studies have proven effective to clear pathological tau species in animal models of AD, and several clinical trials using conventional immunotherapy with anti-tau native antibodies are currently active. We have previously generated a vectorized ScFv derived from the conformation-dependent anti-tau antibody MC1, scFvMC1, and demonstrated that its intracranial injection was able to prevent tau pathology in adult tau mice. Here, we show that, in a prevention paradigm and in two different tau transgenic models (JNPL3 and P301S), a one-time intramuscular injection of AAV1-sCFVMC1 generated a long-lasting peripheral source of anti-tau sCFVMC1 and significantly reduced insoluble and soluble tau species in the brain. Moreover, our data showed that scFvMC1 was internalized by the microglia, in the absence of overt inflammation. This study demonstrates the efficacy of intramuscular delivery of vectorized scFv to target tau, and suggests a new potential application to treat AD and the other tauopathies.
\end{abstract}

Keywords: Vectorized antibodies, AAV, scFv, Tau, Immunotherapy, Intramuscular injection

\section{Introduction}

The microtubule-associated protein tau plays a physiological role in microtubule stabilization, axonal growth and cytoskeletal dynamics in neurons, but its aggregation characterizes several neurological diseases classified as tauopathies, including Alzheimer's disease (AD) [15]. As increasing evidence supports the existence of tau as an extracellular protein and the concept of its transcellular propagation as a mechanism for the initiation and progression of $\mathrm{AD}$, tau has become an attractive target for immunotherapy in animal models of $\mathrm{AD}$ and

\footnotetext{
* Correspondence: Igiliberto@northwell.edu; cdabramo@northwell.edu ${ }^{1}$ Institute of Molecular Medicine, The Litwin-Zucker Center for Alzheimer's Disease \& Memory Disorder, The Feintein Institutes for Medical Research, Manhasset, NY, USA

Full list of author information is available at the end of the article
}

related tauopathies [6-13]. A conspicuous amount of data has been produced by different laboratories, including ours, showing reduction of tau pathology in transgenic animal models using tau monoclonal antibodies, with a different degree of success according to which epitopes were targeted [14-26]. However, passive immunotherapy using conventional antibodies in humans presents several potential limitations such as low permeability to cross the blood-brain barrier (BBB), detrimental inflammatory reactions and microhemorrhages associated to the treatment, requirement for repeated dosing, patients compliance and high costs [27-30]. To overcome these issues, several groups have engineered antibodies as fragments, i.e. single chain variable fragment $(\mathrm{scFv})$, to be used in combination with gene delivery strategies based on the use of viral vectors: 
vectorized anti-Aß (amyloid-beta) and anti-tau scFvs have previously shown benefits in models of AD [3145]. ScFvs consist of the smallest functional antigenbinding domain of an antibody $(\mathrm{Ab})$ exhibiting comparable antigen-binding affinity as the parent immunoglobulin, reduced size, improved pharmacokinetic in terms of tissue penetration and lack of an Fc receptormediated inflammatory response [46-48]. Due to their short systemic half-life in vivo $[49,50]$, in order to reach a sustained and long-lasting expression $\mathrm{scFv}$ are generally cloned in adeno associated viral vectors (AAVs) and delivered by one-time injections [34, 46, 47]. We have previously shown [43] that the AAV vectorizedscFvMC1, a recombinant version of the native anti-tau conformational mAb (monoclonal antibody) MC1, significantly reduces brain pathological tau in adult JNPL3 mice, by one-time intracranial injection. In parallel with AAV-based delivery, Spencer et al. [44] have shown that systemic injection of a lentiviral vector (LV) carrying a scFv directed to 3Rtau and enhanced for brain penetration (LV-3RT-apoB) was able to reduce tau accumulation, neurodegeneration and behavioral deficit in a tau transgenic model. Although using third-generation lentiviral vectors has emerged as a promising therapeutic option for conditions as primary immunodeficiencies and cancers, it is still necessary to understand the long-term safety and efficacy of these vectors in humans, especially in respect to their potential for insertional oncogenesis [51]. In this context, using AAVs is considered safer than LVs.

Direct delivery of AAV into the brain has been tested in a number of clinical trials [52-57] and parallel efforts have been done over the past years to develop new brain-targeted AAVs to treat CNS diseases in vivo using a systemic delivery approach [58]. In this respect, peripheral administration provides obvious advantages to AAV gene therapy: a non-invasive route of injection, lack of surgery-related side effects, improved patient compliance and costs. In this study we propose to develop a novel therapeutic approach for $\mathrm{AD}$ and tauopathies by using intramuscular (IM) delivery of AAVvectorized scFv. To date, there has been little effort to develop peripheral protocols targeting skeletal muscle to tackle CNS disorders. IM injection provides a quick, easy, non-invasive and safe route of administration, and can routinely be performed in virtually any setting. Also, skeletal muscles are an ideal target tissue for AAV transduction because individual fibers are large, multinucleated and with minimal cellular turnover [59]. Taking advantage of the practical features and biological properties of this delivery approach, the aim of the present study was to generate a long-lasting peripheral niche able to produce and release anti-tau $\mathrm{scFv}$ in the circulation to target cerebral tau, without transducing vital organs such as liver, kidneys and heart. A similar strategy has been previously tested in a mouse model of $\mathrm{AD}$, where intramuscular delivery of AAV1 vectored anti-Aß $\mathrm{scFv}$ was able to reduce Aß load in brain [35, 45]. Similarly, gene therapy for Alpha-1 antitrypsin (AAT) deficiency has been developed in humans using recombinant AAV1 serotype, demonstrating a continued stable transgene expression at 5 years after transduction $[60,61]$.

In this study, for the first time, we demonstrate the in vivo feasibility and efficacy of targeting pathological tau in the brain, by employing intramuscular delivery of vectorized anti-tau scFv. Two different tau transgenic models, the JNPL3 and P301S mice, received a single IM injection of AAV1-scFvMC1, showing a significant reduction of tau pathology, with some differences between strains. Moreover, no signs of inflammation were observed upon AAV1-scFvMC1 immunization, showing that tau clearance does not involve Fc-receptormediated and microglia-associated detrimental inflammatory response. However, our in vitro and in vivo data point to the microglia as a player in the uptake and clearance of scFv-tau, despite the absence of Fc, adding to the potential mechanisms of action of tau immunotherapy.

In summary, our data support the peripheral intramuscular route as an effective, feasible and safe delivery approach for AAV-scFv-based anti tau immunotherapy, with relevant translational potential applications to other tauopathies and brain disorders.

\section{Methods}

\section{ScFv-MC1 design and sub-cloning into AAV1}

The light and heavy-chain variable domains corresponding to the $\mathrm{MC} 1$ antibody were sequenced employing the MCLAB antibody service (San Francisco, CA). As previously published [43] the $\mathrm{V}_{\mathrm{H}}$ and $\mathrm{V}_{\mathrm{L}}$ chains were joined together by a 15 amino acid residues linker $\left(\mathrm{Gly}_{4} \mathrm{Ser}\right)_{3}$. 5 '-terminal signal peptide (SP) and 3'-terminal Myc and His6X tags were added. The AAV packaging and purification service was provided by Vector Biolab (Malvern, $\mathrm{PA})$. ScFv-MC1 was sub-cloned into the adenoassociated viral vector serotype 1 (AAV1) under the control of the synthetic strong CAG (CMV-chicken beta actin-rabbit beta globin) promoter. In order to enhance expression of the transgene, the WPRE Woodchuck hepatitis virus (WPRE) post-transcriptional regulatory element was added 5' of the Myc and His6X tags.

\section{Tau transgenic mice}

JNPL3 mice obtained from Taconic (Germantown, NY) express 0N4R human tau with the P301L mutation that causes frontotemporal dementia in humans, under the mouse prion promoter. JNPL3 mice develop NFTs-like 
pathology as early as 4.5 months and in later stages progressive deterioration of the motor function [62]. Homozygous P301S were obtained from Dr. Michel Goedert (Cambridge, UK) [63]: these mice, on pure C57BL/6 background, express 0N4R human tau carrying the P301S mutation, under the control of the neuronspecific murine Thy-1 promoter, and they develop widespread tau pathology affecting cerebral cortex, hippocampus and brain stem as early as 6 months, and partial paralysis of the lower limbs by 8 months of age. Animals were treated according to the current regulations for the proper handling of research animals, following an approved IACUC protocol.

\section{ScFvMC1 purification}

ScFvMC1 purification was performed as previously published [43]. Briefly, scFv-MC1 was cloned into the mammalian expression vector pcDNA3.1 (Genewiz, South Plainfield, NJ) and transfected into HEK293T, using Lipofectamine 2000 (Invitrogen, Carlsbad, CA). After $48 \mathrm{~h}$ of transfection, the $\mathrm{scFv}$ released into the conditioned medium was affinity purified using a Ni-Sepharose High Performance column (GE Healthcare, Port Washington, $\mathrm{NY}$ ). The efficiency of purification was tested using an immunosorbent assay employed to assess the antigenbinding specificity of the scFvMC1, as previously described [43]. Starting material, flow through and eluted fractions were tested to check for proper enrichment of the purified material. The purified $\mathrm{scFv}-\mathrm{MC} 1$ was checked on Coomassie-stained SDS-PAGE gel for proper molecular weight.

\section{Infrared conjugation and intravenous (IV) injections}

$\mathrm{ScFv}-\mathrm{MC1}$ and $\mathrm{MC} 1$ have been conjugated with IRDye $800 \mathrm{CW}$, using IRDye $800 \mathrm{CW}$ protein Labeling Kit LowMW or High-MW respectively (LI-COR Biosciences, Lincoln, NE), according to the manufacturer instructions. Briefly, scFv-MC1 and $\mathrm{MC1}$ were dialyzed in 50 $\mathrm{mM}$ potassium phosphate buffer $\mathrm{pH} 8.5$ at $4{ }^{\circ} \mathrm{C}$, overnight; the $\mathrm{pH}$ was then adjusted with $1 \mathrm{M}$ potassium phosphate buffer to 9 . After $2 \mathrm{~h}$ incubation, the unconjugated dye was removed using desalting spin columns (Zeba Desalt Spin Columns, Thermo Scientific). ScFvMC1 and MC1 antigen-binding reactivities were measured against an MC1 specific peptide [43] by immunosorbent assay, in order to exclude loss of activity upon conjugation.

To verify the ability of scFvMC1 and MC1 to cross the blood brain barrier, IV injection was performed in 3month-old JNPL3 mice using $100 \mu \mathrm{g}$ of the antibodies: saline, scFvMC1-IRDye, MC1-IRDye, or unlabeled antibodies were injected ( $n=3$ per group). Mice were anesthetized with isoflurane and the injections performed retro-orbitally. Mice were sacrificed $2 \mathrm{~h}$ post injection; brains were harvested and dissected into cortex (Ctx), hindbrain (HB) and hippocampus (Hip). Homogenization was performed in 1X RIPA buffer (Thermo Fisher Scientific, Waltham, MA) with the Mini protease inhibitor cocktail (Roche, Indianapolis, IN). Brain samples from each region were spotted on $0.45 \mu \mathrm{m}$ nitrocellulose followed by IR signal acquisition at $789 \mathrm{~nm}$, using Sapphire Biomolecular Imager (Azure Biosystems, Dublin, CA).

\section{Intra-muscular (IM) injections}

AAV1-CAG-scFvMC1 or AAV1-CAG-eGFP were injected at a dose of $2 \times 10^{11} \mathrm{GC}$ per mouse. Each AAV was diluted in PBS at a final volume of $50 \mu \mathrm{l}$, and a onetime intramuscular injection was administrated in the right tibialis. Injections were performed upon anesthesia with isoflurane.

Twenty-six females JNPL3 ( $\mathrm{n}=13$ per group) mice were injected at 3 month of age and sacrificed 4 months later. The P301S line was injected at 2 month of age and sacrificed 4 months later; we used twelve females P301S in total ( $n=6$ per group). Overall, 26 JNPL3 and 12 P301S mice were employed in this study.

\section{Brain extracts and tissues preparation}

Mice were sacrificed by isoflurane overdose, decapitated and processed as described previously [14]. The brain was removed and divided at the midline so that just one half of the brain was dissected for biochemical analysis. Cortex, hippocampus and hindbrain were homogenized separately using an appropriate volume of homogenizing buffer, a solution of Tris-buffered saline (TBS), $\mathrm{pH} 7.4$, containing $10 \mathrm{mM} \quad \mathrm{NaF}, 1 \mathrm{mM} \quad \mathrm{Na}_{3} \mathrm{VO}_{4}$ and $2 \mathrm{mM}$ EGTA, plus the complete Mini protease inhibitor cocktail (Roche). Supernatants were analyzed for protein concentration using DC Protein Assay (Bio-Rad Laboratories, Hercules, CA). Brain homogenates were stored at $-80^{\circ} \mathrm{C}$ and used for separate measurement of soluble and insoluble tau. Soluble tau was measured as heat-stable preparation (hsp) from brain. Hsp were prepared by adding 5\% ß-Mercaptoethanol and $200 \mathrm{mM}$ $\mathrm{NaCl}$ to the brain homogenates. Samples were then heated at $100^{\circ} \mathrm{C}$ for $10 \mathrm{~min}$ and cooled at $4{ }^{\circ} \mathrm{C}$ for 30 $\mathrm{min}$. After centrifuging at $14,000 \mathrm{~g}$ in a table-top microcentrifuge at $4{ }^{\circ} \mathrm{C}$ for $15 \mathrm{~min}$, supernatants were collected and 5X sample buffer (Tris-buffered saline, $\mathrm{pH} 6.8$ containing 4\% SDS, 2\% ß-Mercaptoethanol, 40\% glycerol and $0.1 \%$ bromophenol blue) was added. To obtain insoluble tau preparations (INS), homogenates were thawed and spun at $14,000 \mathrm{~g}$ for $10 \mathrm{~min}$ at $4{ }^{\circ} \mathrm{C}$. The collected supernatants were centrifuged at $200,000 \mathrm{~g}$ for 30 $\min$ at $4{ }^{\circ} \mathrm{C}$; the pellets were then re-suspended in homogenizing buffer and centrifuged again at 200,000 g for $30 \mathrm{~min}$ at $4{ }^{\circ} \mathrm{C}$. The final pellets were re-suspended in $1 \mathrm{X}$ 
sample buffer and heated at $100^{\circ} \mathrm{C}$ for $10 \mathrm{~min}$ to efficiently dissociate the insoluble tau fraction.

Liver, kidney and heart were harvest and homogenated using 1X RIPA buffer with the complete Mini protease inhibitor cocktail (Roche). Protein concentration were analyzed using DC Protein Assay (Bio-Rad Laboratories) and samples were prepared for western blotting. For tibialis and gluteus maximum muscles protein were extracted in skeletal muscle homogenizing buffer $(20 \mathrm{mM}$ Tris, $137 \mathrm{mM} \mathrm{NaCl}, 2.7 \mathrm{mM} \mathrm{KCl}, 1 \mathrm{mM} \mathrm{MgCl} 2,1 \%$ Triton X-100, $10 \%$ glycerol, $1 \mathrm{mM}$ EDTA and $1 \mathrm{mM}$ dithiothreitol) plus the complete Mini protease inhibitor cocktail (Roche). Tissue were mince using a Douncehomogenizer, sonicated and then let vortexed overnight at $4{ }^{\circ} \mathrm{C}$. The supernatant, containing the protein extract, was collected after $15 \mathrm{~min}$ centrifugation at $14,000 \mathrm{~g}$ and used in immunoblot, as described later.

\section{Tau ELISA}

Levels of total and phosphorylated tau were assessed using the Low-tau ELISA (enzyme-linked immunosorbent assay) protocol previously published [64, 65]. 96-well plates were coated for $48 \mathrm{~h}$ at $4{ }^{\circ} \mathrm{C}$ with specific purified monoclonal tau antibodies (DA31, CP13, PHF1, RZ3) at a concentration of $6 \mu \mathrm{g} / \mathrm{ml}$. After washing, plates were blocked for $1 \mathrm{~h}$ at RT using StartingBlock buffer (Thermo Fisher Scientific). Brain samples and standards were diluted in 20\% SuperBlock buffer (Thermo Fisher Scientific) in 1XTBS and loaded on the plates. Once the samples were added, the total tau detection antibody DA9-HRP, diluted 1:50 in 20\% SuperBlock in 1XTBS, was added to the samples and tapped to combine. Plates were then incubated overnight at $4{ }^{\circ} \mathrm{C}$. Next day, 1-Step ULTRA TMB-ELISA (Thermo Fisher Scientific) was added for $30 \mathrm{~min}$ at $\mathrm{RT}$, followed by $2 \mathrm{~N} \mathrm{H}_{2} \mathrm{SO}_{4}$ to stop the reaction. Plates were read with Infinite $\mathrm{m} 200$ plate reader (Tecan, San Jose, CA) at $450 \mathrm{~nm}$.

\section{Immunoblotting}

An aliquot of the total lysates was used for western blotting (WB). 0.1\% SDS was added to the lysates, followed by sonication ( 3 cycles, $10 \mathrm{~s}$ each). Samples were run on 4-20\% Criterion Tris- $\mathrm{HCl}$ gels (Bio-Rad Laboratories) and electrophoretically transferred to a nitrocellulose membrane (Thermo Fisher Scientific). Residual proteinbinding sites were blocked by incubation with $5 \%$ nonfat milk in 1XTBST (1X TBS plus $0.1 \%$ Tween 20 ) $1 \mathrm{~h}$ at $\mathrm{RT}$, followed by an overnight $(\mathrm{O} / \mathrm{N})$ incubation at $4{ }^{\circ} \mathrm{C}$ with primary antibodies diluted in 20\% SuperBlock buffer (Thermo Fisher Scientific) in 1XTBST. Mouse antitubulin (Thermo Fisher Scientific) were diluted 1:5000; anti-Myc-tag 9B11 (Cell Signaling, Danvers, MA) was diluted 1:1000. Appropriate isotypes secondary antibodies HRP-conjugated were diluted $1: 2000$ or 1:10000 in 5\% non-fat milk 1XTBST, and added for $1 \mathrm{~h}$ at RT. Every step was followed by 3 or 4 washes in 1X TBST. Detection was performed using Pierce ECL Western Blotting Substrate (Thermo Fisher Scientific) or SuperSignal West Dura extended duration substrate (Thermo Fisher Scientific) and exposed to $\mathrm{x}$-ray films.

\section{Immunocytochemistry, immunofluorescence and image analysis}

Tau staining and immunofluorescence were performed according to standardized protocols [14, 43]. After decapitation, half of the brain was fixed overnight in $4 \%$ paraformaldehyde at $4{ }^{\circ} \mathrm{C}$. Serial sections were cut from the fixed brain half on a vibratome, conserved in TBS (50 mM Tris, $150 \mathrm{mM} \mathrm{NaCl}, \mathrm{pH} 7.6$ )/0.01\% $\mathrm{NaN}_{3}$, and stained on 24-well plates with a panel of tau antibodies. Endogenous peroxidases were quenched with $3 \% \mathrm{H}_{2} \mathrm{O}_{2}$ / $0.25 \%$ Triton X-100/1XTBS for $30 \mathrm{~min}$. Non-specific binding was blocked with 5\% non-fat milk-1XTBS for 1 $\mathrm{h}$ at RT. Primary antibodies were used as follows: anti tau antibodies RZ3 and MC1 (1:500), CP13 and PHF1 (1:5000); all antibodies were diluted in $5 \%$ non-fat milk1XTBS, and incubated $\mathrm{O} / \mathrm{N}$ at $4{ }^{\circ} \mathrm{C}$, shaking. Biotinconjugated secondary antibodies (SouthernBiotech, Birmingham, AL) directed against the specific isotypes were diluted 1:1000 in 20\% SuperBlock, left for $2 \mathrm{~h}$ at RT, and lately Streptavidin-HRP (SouthernBiotech) was incubated for $1 \mathrm{~h}$. Staining was visualized by 3,3'-Diaminobenzidine (Sigma-Aldrich, St. Louis, MO). Images were acquired using Olympus BH-2 bright field microscope (Waltham, MA); analyzed and processed using ImageJ/ Fiji software (NIH). Semi-quantification was done on the hippocampal quadrant CA1 and on the entorhinal cortex by using the measure particles tool, working with 8-bit images and adjusting the threshold.

For immunofluorescence, sections were pre-incubated 5 min at RT in 1XTBS (Gibco, Carlsbad, CA) containing $0.2 \%$ TritonX100 (Sigma-Aldrich). After blocking $1 \mathrm{~h}$ at RT with a solution containing $5 \%$ normal goat serum (Sigma-Aldrich) diluted in 1XTBS/0.2\% Triton, sections were incubated with primary antibodies diluted in $1 \%$ normal goat serum in 1 XTBS $/ 0.2 \%$ Triton: anti-MycAlexa Flour555 1:500 (EMD Millipore), Iba-1 1:1000 (Wako Chemicals, Richmond, VA), anti-CD68 1:200 (Bio-Rad Laboratories) and RZ3 (anti-tau pThr231) 1: 500. After washing $3 \mathrm{X}$ in $1 \mathrm{XTBS} / 0.2 \%$ TritonX100, Alexa Fluor secondary antibodies -488 and -568 and 350 (Invitrogen) were added at 1:1000 or 1:2000 dilutions for $1 \mathrm{~h}$ at RT, in different combinations in order to obtain multiple labeling images. DAPI (Invitrogen) was used to counterstain. Brains slices were then mounted on slides and let dry $20 \mathrm{~min}$ before being coverslipped using Vectashield hard set anti-fade mounting (Vector Laboratories, Burlingame, CA). Sections incubated 
without primary antibody were used as negative controls. Images were acquired using Zeiss 880 confocal laser microscope (Peabody, MA). Integrated intensity was quantified using $\mathrm{NIH}$ Image $(\mathrm{NIH})$ on raw images, with background fluorescence subtraction on predefined ROIs.

For Iba-1 VIP-substrate staining (Vector Laboratories, Burlingame, CA), antigen retrieval was performed using 1X Dako Target Retrieval solution (Agilent Dako, Santa Clara, CA, USA) in distilled water $/ 0.5 \%$ Triton, at $95-$ $99^{\circ} \mathrm{C}$ for $5 \mathrm{~min}$. After washing, endogenous peroxidases were quenched with $3 \% \quad \mathrm{H}_{2} \mathrm{O}_{2} / 0.25 \%$ Triton X-100/ 1XTBS for $30 \mathrm{~min}$. Sections were incubated in $5 \%$ normal goat serum (Sigma Aldrich) in 1XTBS/0.1\% Triton. Primary polyclonal antibody, anti Iba-1 (Wako Chemicals, Richmond, VA), was diluted 1:2000 in 1\% normal goat serum in $1 \mathrm{XTBS} / 0.1 \%$ Triton and let incubate $\mathrm{O} / \mathrm{N}$ at $4{ }^{\circ} \mathrm{C}$. Biotin-conjugated goat anti-rabbit secondary antibody (SouthernBiotech) were used at 1:2000 in 20\% SuperBlock (ThermoFisher) in $1 \mathrm{X}$ TBS/0.05\% Triton, left for $2 \mathrm{~h}$ at RT, and lately Streptavidin-HRP (SouthernBiotech) was incubated for $1 \mathrm{~h}$. Staining was visualized using Vector VIP Substrate (Vector Laboratories) following the manufacture's specifications. After washing with distilled water slides were mounted and coverslipped. Microglia were imaged on AxioImager Z1 microscope (Zeiss) at 63x oil and $0.58 \mu \mathrm{m} \mathrm{z}$-steps to capture 3 ROIs across the stratum radiatum of the CA1 subfield of the hippocampus for each animal (5 mice per group, 10 cells per mouse imaged: 50 cells per treatment group analysed). The microglia process morphology was categorized with a score from 0 to 3, following the criteria described by Schafer et al. [66-68]: 0 (>15 thin processes with multiple branches), 1 (5-15 thick processes with branches), 2 (1-5 thick processes with few branches), 3 (no clear processes). All analyses were performed in blind.

On peripheral organs, histology was performed by HistoWiz Inc. (histowiz.com) using a Standard Operating Procedure and fully automated workflow. Samples were processed, embedded in paraffin, and sectioned at $4 \mu \mathrm{m}$. Immunohistochemistry was performed on a Bond $\mathrm{Rx}$ autostainer (Leica Biosystems) with enzyme treatment (1:1000) using standard protocols. Slides were stained with hematoxylin and eosin and anti-NFkb. Bond Polymer Refine Detection (Leica Biosystems) was used according to manufacturer's protocol. After staining, sections were dehydrated and film coverslipped using a TissueTek-Prisma and Coverslipper (Sakura). Whole slide scanning (40X) was performed on an Aperio AT2 (Leica Biosystems).

Primary mouse microglia cultures and uptake experiment Cultures were prepared from post-natal C57BL/6 mouse pups at 2 days of age. Whole brains were trypsin digested and made into a cell suspension. Cells were seeded in flasks pre-coated with $0.1 \mathrm{mg} / \mathrm{ml}$ poly-D-lysine (Sigma-Aldrich) and maintained in DMEM supplemented with $10 \%$ heat-inactivated FBS (Gibco) and 1\% Pen-Strep (Gibco). Medium was supplemented with 5 $\mathrm{ng} / \mathrm{ml}$ Macrophage Colony Stimulating factor (M-CSF) (Thermo Fisher Scientific) diluted in PBS supplemented with $0.1 \%$ sterile filtered BSA (Sigma-Aldrich). At DIV10 microglia were isolated by orbital shaking at 150 RPM for $1 \mathrm{~h}$ and the supernatant was seeded in 12-well plates with 300,000 cells per well. Experiments were performed on the subsequent day. PHF-tau (paired helical filaments) [69] was added to microglia at a concentration of $1 \mu \mathrm{g} / \mathrm{ml}$ as determined by total tau ELISA. ScFvMC1 was added at a concentration of $10 \mu \mathrm{g} / \mathrm{ml}$. To allow for immune complex formation, PHF-tau and scFvMC1 were mixed in medium and pre-incubated at $37^{\circ} \mathrm{C}$ for $30-45$ min prior to addition to cells. Mixing was performed two times during incubation by repeated manual pipetting. The $2 \mathrm{~h}$ incubation was performed in medium without serum. All experiments were performed in triplicate, with each treatment group in quadruplicate. The amount of PHF-tau in medium at the end of the experiments was assessed using the same low-tau ELISA previously described.

\section{Stereotaxic intracranial injection}

Intra-hippocampal injections of AAV vectors were performed according to a stereotaxic surgery protocol previously published [43]. Briefly, under sterile conditions, 3-month-old P301S mice were anesthetized and secured on a stereotaxic frame (David Kopf instruments, Tujunga, CA). Mice received bilateral hippocampal injection of AAV preparations using a neuro syringe with a 33 gauge needle (Hamilton, Reno, NV), using the following coordinates: AP -2.1 from bregma, $\mathrm{ML}+/-2.0$ from bregma, DV -1.8 below dura. Animals were treated according to the current regulations for the proper handling of research animals, following an approved IACUC protocol.

\section{Flow cytometry on adult mice microglia}

Microglia was isolated from 6-month-old P301S mice treated with AAV5-scFv-MC1 and AAV5-null injected mice. In this experiment, we used an AAV-null construct instead of AAV-eGFP, since our goal was to ascertain the uptake of the scFv by microglia and since eGFP may interfere with flow cytometry analysis. Mice were anesthetized and cold PBS-perfused. After dissection, the forebrain was minced with a Dounce homogenizer in ice cold HBSS, filtered onto $70 \mu \mathrm{m}$ cell strainer, and centrifuged at $300 \mathrm{~g}$ for $5 \mathrm{~min}$ at $4{ }^{\circ} \mathrm{C}$. Tissue was dissociated using Neural Dissociation Kit P (MACS Miltenyi Biotec, Auburn CA) according to the manufacturer's 
instruction. Myelin debris were removed using Myelin Removal Beads II (MACS Miltenyi Biotec). Briefly, after neural dissociation, samples were spun at $300 \mathrm{~g}$ for 10 min at $4{ }^{\circ} \mathrm{C}$ and incubated 15 min with Myelin Removal Beads in 0.5\% BSA in 1X PBS. Cells suspension was then loaded onto a pre-washed MACS LS column and placed in the magnetic field of MACS Separator. The magnetically labeled myelin was retained within the column while unlabeled cells run through [70]. Cells suspension, myelin depleted, was then washed twice with FACS buffer (0.05\% BSA, $0.02 \%$ sodium azide in $1 \mathrm{X}$ PBS) and stained with Live/Dead-Pacific Blue (Thermo Fisher Scientific). Surface staining was performed using CD11b-PE and CD45-APC/Cy7 antibodies (BD Biosciences, Franklin Lakes, NJ) in order to select microglia from other monocytes. After fixation and permeabilization with BD Cytofix/Cytoperm Fixation/Permeabilization Solution Kit (BD Biosciences) cells were stained with anti-Myc Tag Antibody AlexaFluor-647 (Thermo Fisher Scientific). Debris, doublets and dead cells were excluded using fsc/ ssc, fsc-h/fsc-w and Pacific Blue gates, respectively. BD CompBeads (BD Biosciences) were used for calibration of flow cytometer. Samples were analyzed on a BD LSRFortessa and data processed using FlowJo software (Treestar).

\section{Tau and anti-scFvMC1 antibodies detection in serum}

A detailed protocol was previously published to detect total tau in serum [71]. Upon sacrifice mice were bled, samples collected and allowed to clot for $30 \mathrm{~min}$ at RT. After cooling for $15 \mathrm{~min}$, samples were spun at $14,000 \mathrm{~g}$ for $10 \mathrm{~min}$ at $4{ }^{\circ} \mathrm{C}$; supernatants were collected and then re-spun at $14,000 \mathrm{~g}$ for $5 \mathrm{~min}$ at $4{ }^{\circ} \mathrm{C}$. The final supernatants correspond to the serum samples. In order to detect tau in serum, samples were diluted $1: 3$ in $0.2 \mathrm{M}$ $\mathrm{NaOAc}, \mathrm{pH} 5.0$ and heated at $90^{\circ} \mathrm{C}$ for $15 \mathrm{~min}$. After the heat treatment, samples were allowed to cool at $4{ }^{\circ} \mathrm{C}$ for $15 \mathrm{~min}$, and then spun at $15,000 \mathrm{~g}$ for $10 \mathrm{~min}$. Supernatants were collected and $1 \mathrm{M}$ Tris buffer was added to neutralize the $\mathrm{pH}$. After diluting 1:2 in 20\% Superblock, samples were loaded on the total tau ELISA (DA31 capture).

In order to detect antibodies directed to the scFvMC1, 96-well plates were coated with purified scFvMC1 at $6 \mu \mathrm{g} / \mathrm{ml}$ for at least $24 \mathrm{~h}$. Plates were washed $3 \mathrm{X}$ and blocked for $1 \mathrm{~h}$ using StartingBlock (Thermo Fisher Scientific). Plates were washed $5 \mathrm{X}$ and $50 \mu \mathrm{l}$ of sera added in triplicate at 1:1000 dilution in 20\% SuperBlock (Thermo Fisher Scientific). After $1 \mathrm{~h}$ incubation plates were washed $5 \mathrm{X}$ and $50 \mu \mathrm{l}$ of goat anti-mouse nonspecific IgG HRP-conjugated (SoutherBiotech, Birmingha, AL) was added and incubated for $1 \mathrm{~h}$. Finally, BioRad HRP Substrate Kit has been used for the detection and plates were read with Infinite m200 plate reader (Tecan) at $415 \mathrm{~nm}$.

\section{Statistical analysis}

Quantitative data were analyzed using the dedicated software GraphPad Prism V.6 (GraphPad software Inc., CA). Unpaired t test with Welch's correction was performed when the parametric assumption of normality (D'Agostino-Pearson omnibus test) was accomplished. When not, non parametric Mann-Whitney test was performed instead. Statistical significance was set at $P<$ 0.05 . Error bars represent the standard error of the mean (SEM).

\section{Results}

ScFvMC1 is detected in the brain homogenates upon intravenous injection of the purified SCFv

We first asked whether scFvMC1 crosses the BBB and targets the brain [72, 73]. After intra-peritoneal (IP) peripheral injection, we were not able to detect brain scFvMC1 using antibodies directed against Myc or 6-His (tags present on our scFv construct), via IHC, WB or ELISA (not shown). We have thus performed a proof-of concept experiment, testing $\mathrm{BBB}$ penetration via retroorbital IV injection of $100 \mu \mathrm{g}$ of purified scFvMC1, either unlabeled (UNL) or infrared-conjugated (IR), in adult JNPL3 and P301S mice; a careful comparison was performed between scFvs and native $\mathrm{MC} 1$ variants, including saline as a negative control. Given the scFv's short half-life, mice were sacrificed $2 \mathrm{~h}$ post-injection and perfused with ice-cold PBS/Heparin. The serum concentration of both scFvMC1-IR and the native MC1-IR were calculated in the amount of $50 \mathrm{ng} / \mu \mathrm{l}$. At termination of the experiment, scFvMC1 was detected in cortex, HB and hippocampus (Fig. 1a): its amount was in the range of $0.1-0.2 \%$ of the serum concentration, in line with the current literature [40, 72, 74, 75], confirming the feasibility of a peripheral approach that relies on a sustained release of $\mathrm{scFv}$ in the circulation.

\section{AAV1-scFvMC1 specifically transduces muscle cells upon one-time intramuscular injection}

Since scFvMC1 efficiently crosses the BBB, we have next focused on selecting a peripheral tissue to be transduced by the AAV-scFv construct, to generate a stable source of antibody, with the goal to circumvent vital organs. Skeletal muscle is considered an ideal target tissue for AAV transduction because individual fibers are large, multinucleated and with minimal cellular turnover [35, 59, 76]. Hence, we have selected the AAV1 serotype to produce a stable muscular niche able to continuously produce anti-tau scFvMC1 and release it into the circulation to target cerebral tau. In a prevention protocol, given the different timeline in the development of tau 


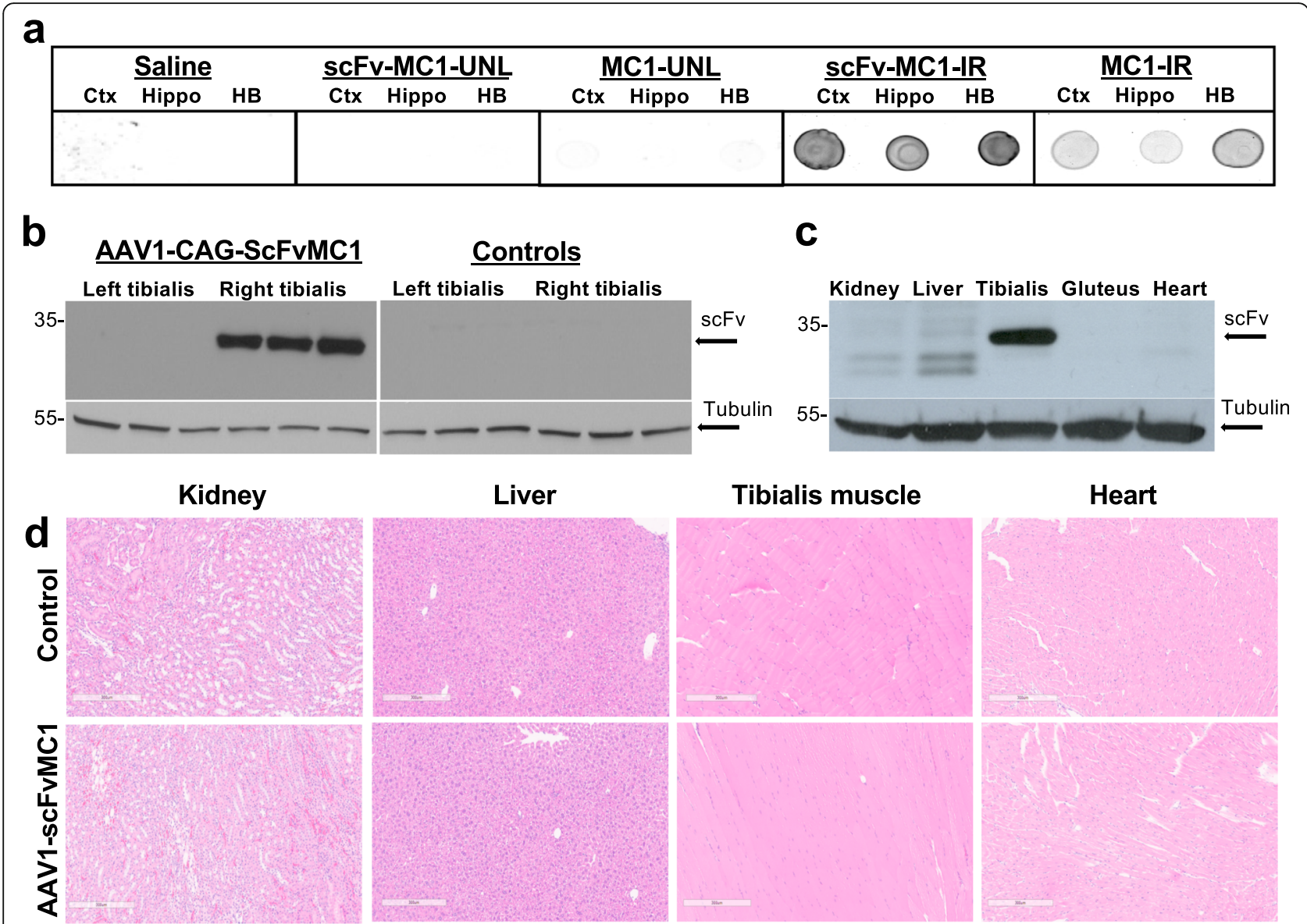

Fig. 1 SCFVMC1 detection and peripheral organs' morphology. a Six-month old mice (JNPL3 shown) were IV-injected with saline, $100 \mu \mathrm{g}$ of IRDye-labelled antibodies (scFvMC1-IR and MC1-IR) and unlabeled antibodies (scFvMC1-UNL and MC1-UNL). Two hours post-injection, brain homogenates from cortex (Ctx), hippocampus (Hip) and hindbrain (HB) were spotted on nitrocellulose and absorbance of the IRDye label measured. Saline and unlabeled antibodies showed no signal (Sapphire Biomolecular Imager, Azure Biosystems). b, c AAV1-CAG-scFvMC1 was injected in tibialis muscle of P301S or JNPL3 (JNPL3 shown), mice sacrificed 4 months later and peripheral organs harvested. Tissue lysates were analyzed for scFvMC1 expression with anti-Myc/tag antibody showing localized expression in the injected site (b, right tibialis, Controls = AAV1eGFP) while other peripheral organs did not show scFv signal (c). ScFvMC1: MW around 30 kDa; tubulin is used as housekeeper: MW at 55 kDa. d Hematoxylin and eosin (H\&E) staining was performed on kidney, liver, tibialis muscle and heart: representative images of each organs show no significant changes in morphology (Controls = AAV1-CAG-eGFP; Bright field microscope, scale bar: $300 \mu \mathrm{m}$ )

pathology in the two mice models selected, 3-month-old JNPL3 and 2-month-old P301S mice were injected in the right tibialis anterior muscles with AAV1-CAGscFvMC1 and sacrificed 4 months post-injection, at 7 or 6 months of age respectively. To ascertain transduction of our AAV system, transgene expression in the injected site was assessed by immunoblotting. Figure $1 \mathrm{~b}$ shows a specific band around $30-35 \mathrm{kDa}$ corresponding to scFv$\mathrm{MC} 1$ expression in the injected tibialis. Other peripheral organs failed to show expression of scFv-MC1 (Fig. 1c), confirming targeted local delivery and the high specificity of the AAV1-CAG system. No premature death or body weight loss were reported throughout the study. Moreover, no changes in tissues morphology at the time of sacrifice were detected as assessed by hematoxylin and eosin (H\&E) (Fig. 1d); the inflammatory state, also unchanged, was assessed using NF-kB staining (Supplementary Fig. 1).

\section{AAV1-scFvMC1 intramuscular injection significantly decreases the insoluble tau burden in both P301S and JNPL3 mice}

To rigorously quantitate the tau burden, we performed an extensive biochemical analysis of insoluble, soluble and oligomeric tau species in brain. We monitored the tau insoluble burden (a proxy for tau aggregation) in cortex and hindbrain by low-tau ELISA (Fig. 2, Fig. 3). As shown in Fig. 2a, we observed a dramatic decrease of cortical insoluble tau in the P301S cohort upon treatment with scFvMC1: total tau $(-70 \%, " * p<0.01)$, pThr231 $\left(-70 \%,{ }^{* * *} p<0.01\right)$, pSer202 $(-60 \%, " p<0.05)$ and pSer396/404 $(-65 \%, " * p<0.01)$. The same 

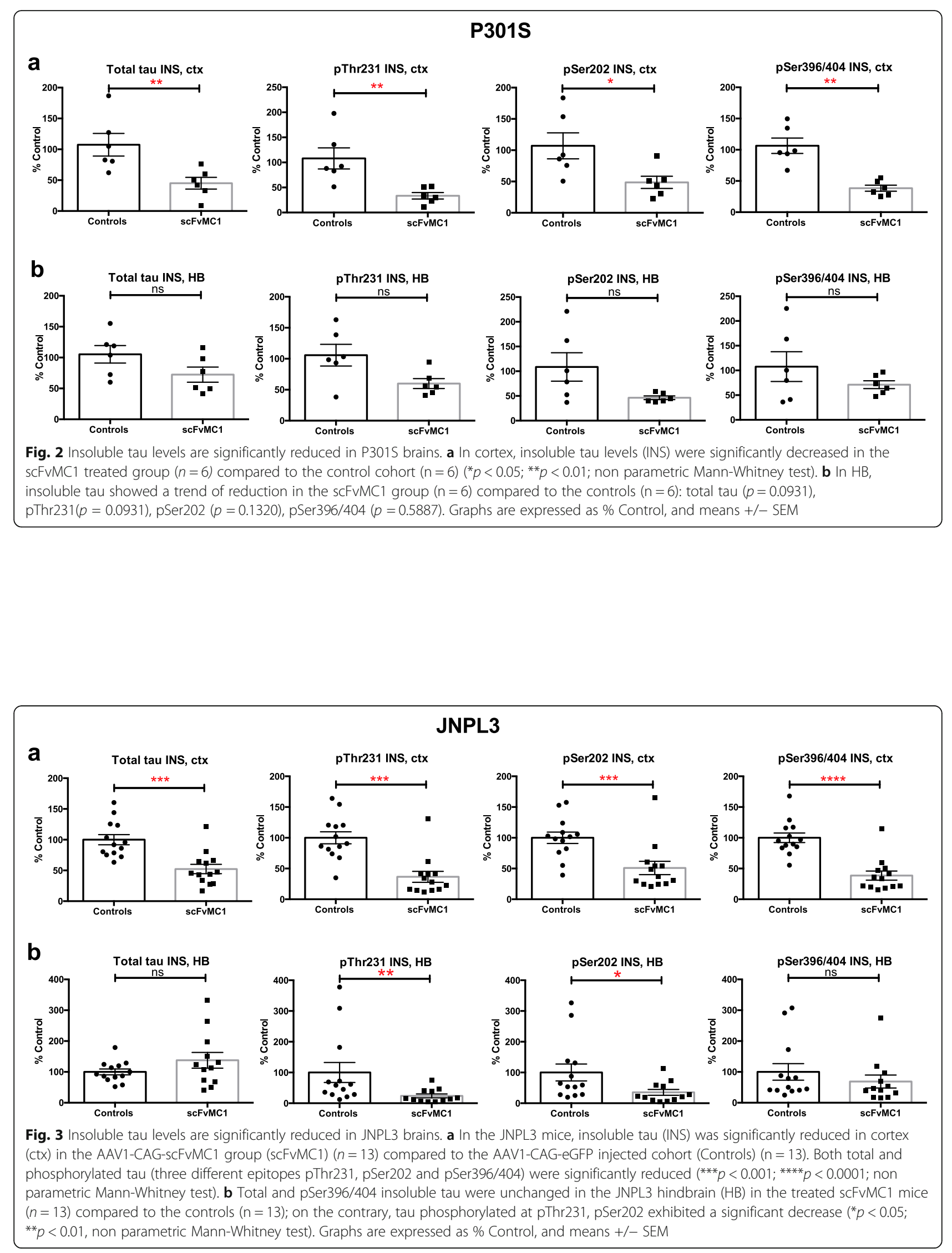
significant reduction was confirmed in the JNPL3 (Fig. 3a) following a sustained peripheral release of scFvMC1, both as total tau $(-50 \%, * * * 0.001)$ and phosphorylation at Thr231 $\left(-65 \%,{ }^{* * * *} p<0.001\right)$, Ser202 $(-50 \%$, $\left.{ }^{* * * * *} p<0.001\right)$ and Ser396/404 $\left(-60 \%\right.$, $\left.{ }^{* * * * * *} p<0.0001\right)$.

Similar to what we observed in cortex, in the P301S mice a trend towards reduction was clearly visible in treated mice's hindbrains (Fig. 2b): $30 \%$ reduction of insoluble total tau in hindbrain was detected although this was not significant at the $p=0.05$ level $(p=0.0931)$; also, 40 and $50 \%$ trends to reduction were detected for pThr231 $(p=0.0931)$ and pSer202 $(p=0.1320)$, respectively. The JNPL3 treated mice's hindbrains exhibited a significant reduction of pThr231 $(-75 \%, * * p<0.01)$ and pSer202 $(-65 \%, " p<0.05)$, while total and tau phosphorylated at Ser396/404 were not decreased (Fig. 3b).

\section{Tau soluble and oligomeric species' levels show strain differences upon treatment}

We next assessed soluble tau from cortex, hindbrain and hippocampus homogenates. Biochemical analysis of soluble tau showed differences between the two mice strains. Analysis of the P301S mice (Fig. 4) showed total and phosphorylated tau soluble species to be significantly reduced in all cerebral regions upon scFvMC1 treatment (Fig. 4a-c; $40-50 \%$ reduction, " $p<0.05$; *" $p<$ 0.01). In contrast, in the JNPL3 mice, we did not observe any modulation of total, pThr231, pSer202 or pSer396/ 404 soluble tau in both cortex and hippocampus (Fig. 5a, c); surprisingly, in the hindbrain, tau phosphorylated at Ser202 and Ser396/404 was augmented in the scFvMC1injected group in JNPL3 mice (Fig. 5b).

Finally, oligomeric/aggregated tau was examined using a tau-mono-ELISA previously developed in our laboratory [65]. This assay, a functional surrogate of insoluble tau measurement, allows the detection of aggregated tau species, ranging from dimers to larger aggregates, and represents a good marker of progression towards the formation of neurofibrillary pathology in tau transgenic animal models. We routinely employ this assay for the measurement of hippocampal aggregated tau. While JNPL3 mice failed again to show any changes between controls and treated mice in all brain areas (Fig. 5d), P301S mice exhibited a significant reduction of oligomeric/aggregated tau species in the hippocampus ($50 \%, " p<0.05)$ and a trend towards reduction in cortex and HB (Fig. 4d), consistent with the data reported above for soluble and insoluble tau.

\section{Tau burden changes are not detected by immunohistochemistry}

In addition we performed an immunohistochemical analysis on JNPL3 (Fig. 6A a-f; Fig. 6B a-f) and P301S brain slices (Fig. 6A g-l; Fig. 6B g-l), by assessing tau pathology in the CA1 hippocampal pyramidal cell layer and in the entorhinal cortex (EC). Semi-quantification of tau phosphorylated at Thr231 and MC1-tau was performed on both CA1 (Fig. 6A c, i; Fig. 6B c, i) and EC (Fig. 6A f, l; Fig. 6B f, l). No significant changes in immunoreactivity were observed, except some trends towards decreased staining in the hippocampal region of JNPL3 mice (Fig. 6 A c, $p=0.1128$; Fig. 6 B c, $p=$ 0.0996). Tau phosphorylation at Ser396/404 and Ser202 (not shown) also failed to show significant reduction when comparing treated to non-treated animals.

\section{Microglia are not activated, but have the ability to uptake the tau-scFvMC1 immunocomplex}

We next sought to determine whether microglia were activated in our system. Confocal microscopy was performed on brains from P301S (Fig. 7a, b) and JNPL3 (Fig. 7c, d) mice to assess the immunoreactivity of two widely studied markers for microglia activation: Iba1 (morphology/activation) and CD68 (reactive/phagocytic). We observed no difference between controls and scFvMC1 treated mice and confirmed these data by semi-quantitative analysis in the stratum radiatum of both strains. Cortex and HB were imaged and quantified displaying similar results (not shown).

To examine deeper the microglia activation state, a morphological assessment was performed (Fig. 8a-c), using a protocol previously described [66], confirming that neither P301S or JNPL3 treated mice exhibited any significant changes in microglia morphology compared to their respective controls.

Given the peripheral injection and the lack of Fc region in the scFv structure, we did not expect an inflammatory response dependent on the microglia Fc receptor (FcR) engagement in our model, but we assumed that the altered functional state of the microglia found in neurodegenerative disorders and tau mouse models [7780] may still facilitate microglia-mediated tau clearance upon scFv treatment. Hence, to deepen our understanding on the microglia's role in our system, we first set out a cell-based proof of concept experiment to determine whether primary microglia could uptake the tauscFvMC1 immunocomplex. Primary microglia were incubated for $2 \mathrm{~h}$ with PHF-tau (paired helical filament, prepared in our lab $[69,81]$ ) with and without scFvMC1 (scFv/PHF ratio 10/1) (Fig. 9). In order to work in the appropriate experimental conditions, PHF-tau and PHFtau/scFvMC1 immunocomplex were applied on plastic plates (- primary microglia) or on microglia seeded plates (+ primary microglia). Primary microglia showed an innate ability to uptake PHF from the medium (Fig. $9 \mathrm{a}, \mathrm{A}$ vs C: $10 \%$ reduction, $" p=0.0168)$, which was enhanced by the presence of scFv-MC1: a $20 \%$ reduction (Fig. 9a, C vs D, " $p=0.0137$ ) was detected when co- 


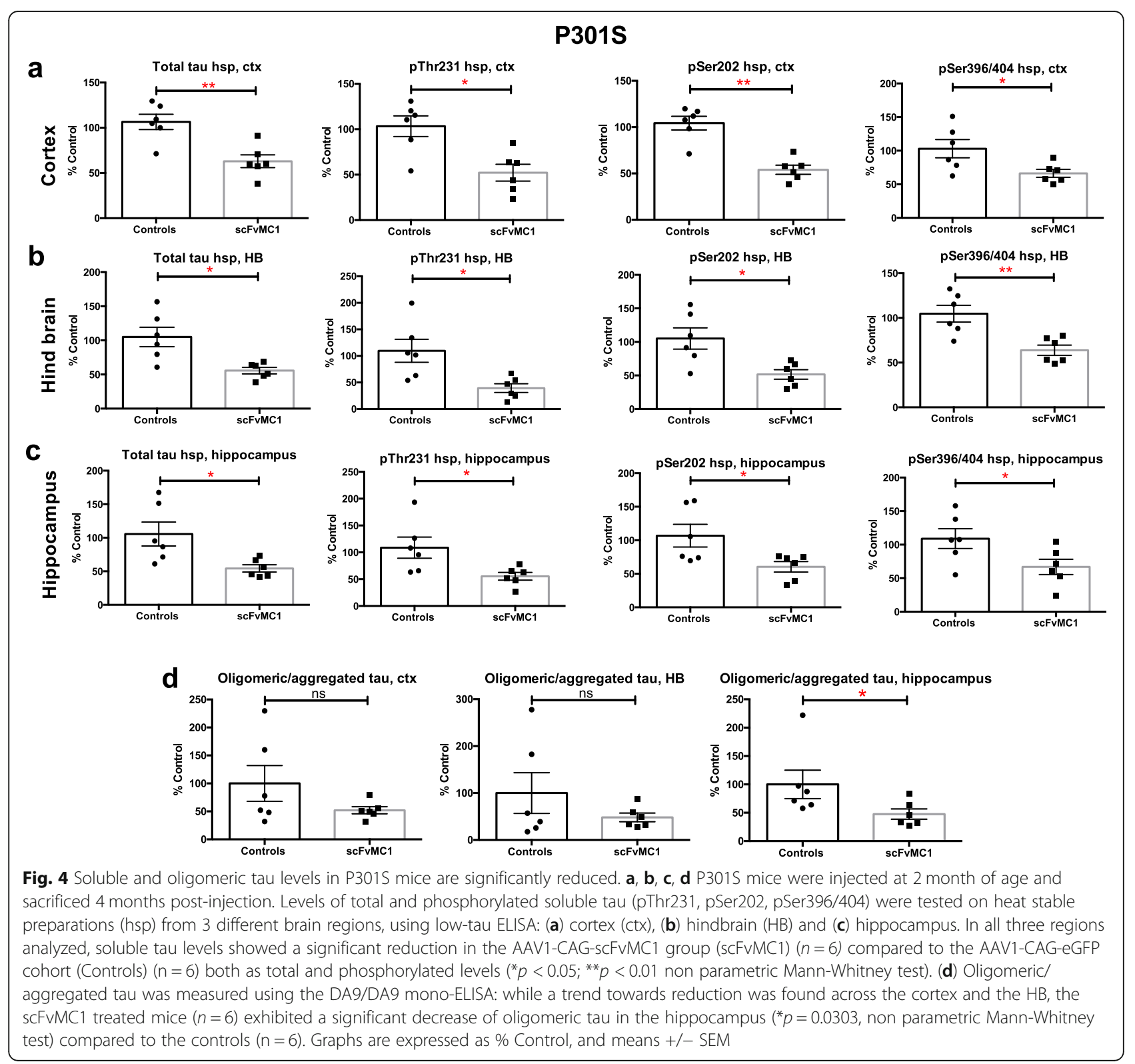

treating the microglia with scFvMC1/PHF compared to PHF alone. Also, in the co-treatment paradigm we observed a $25 \%$ reduction of PHF in medium (Fig. 9a, B vs D, ** $p=$ 0.0019) when working on microglia seeded plates compared to empty plates. Interestingly, intracellular PHF-tau readily disappeared, after uptake, from the microglia lysates when scFvMC1 was present (Fig. 9b, upper panel). Moreover, the levels of scFvMC1 were unchanged in microglia lysates, with and without PHF, suggesting an excess of $\mathrm{scFv}$ in this in vitro experiment (Fig. 9b, lower panel).

Since we were not able to directly visualize scFvMC1 in the brain upon IM injection of the vectorized antibody, to further validate our in vitro data we have utilized adult P301S mice injected intracranially with AAV5-GFAP-scFvMC1, using the same strategy applied in our previous study [43]. Confocal microscopy was performed to co-localize tau and scFvMC1 in microglia confirming that both phospho-tau (pThr231) and scFvMC1 (Myc-555) co-localize in Iba1 positive microglia (Supplementary Fig. 2). Using the same experimental model, we show by confocal microscopy (Fig. $10 \mathrm{~A}$ ) that scFvMC1 is detected in Iba1 positive microglia from treated animals, confirming a role of this cellular population in the uptake and clearance of pathological tau. Finally, adult P301S intracranially injected with AAV5GFAP-scFvMC1 were employed to isolate microglia [70, $77,82,83$ ] from whole brain. Following microglia sorting with flow cytometry (CD11b ${ }^{\text {high }} \mathrm{CD} 45^{\text {low }}$ ) (Fig. $10 \mathrm{~B}$, ad), cells were permeabilized to detect scFvMC1 inside the microglia (anti-Myc-647, blue) in the AAV5- 


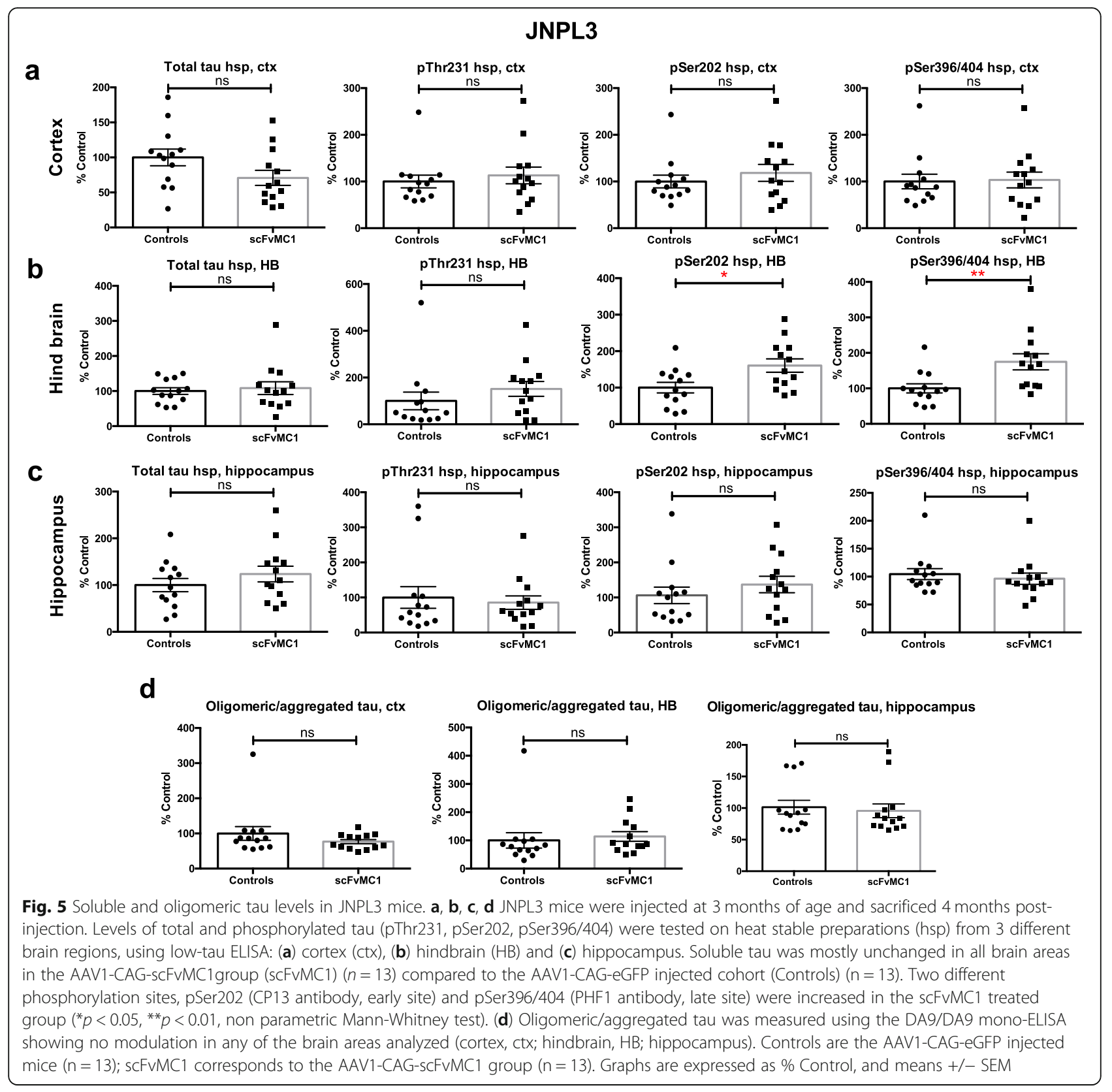

scFvMC1 injected mice; no anti-Myc-647 was detected in the AAV-null injected animals (red) (Fig. $10 \mathrm{~B}, \mathrm{e}$ ).

Overall, our in vitro and in vivo data indicate that, despite the lack of Fc effector function, microglia have the ability to uptake tau and scFvMC1.

\section{Antibodies directed to the scFvMC1 are detected in the JNPL3 mice serum}

A major concern about the long-term use of antibodies as a treatment is the generation of neutralizing antibodies (NAB), which would compromise the therapeutic effect. We have investigated whether expression of $\mathrm{scFv}$ gene in the body would trigger the production of antibodies directed against scFv. Upon sacrifice, serum was collected and processed to test for the presence of scFvMC1 in the circulation. While we failed to directly detect ScFvMC1 in serum, we were able to detect antibodies directed to scFvMC1 in the JNPL3 treated cohort (Supplementary Fig. 3b), similar to what observed in our previous study upon intracranial injection of AAV5-scFvMC1 [43]. Contrarily, the treated P301S mice did not show any detectable anti-scFvMC1 in serum (Supplementary Fig. 3a).

Our serological assessment was completed by determining the tau levels in the circulation, to investigate the ability of scFvMC1 to export tau from the brain parenchyma to the periphery [84]. As shown in 


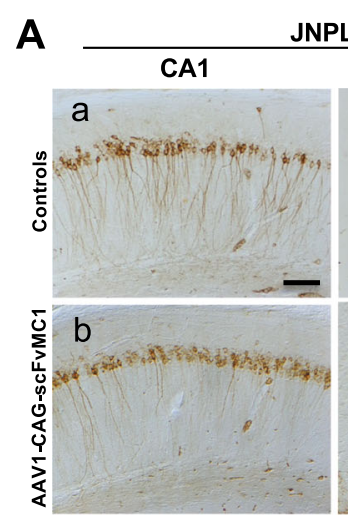

\section{PL3}

EC
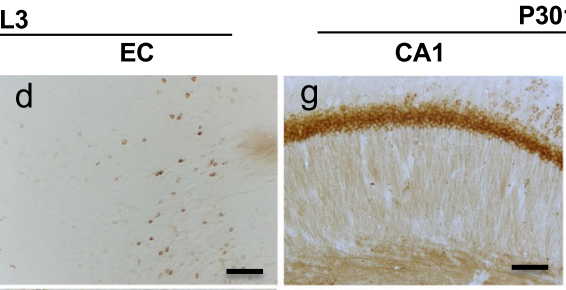

P301S
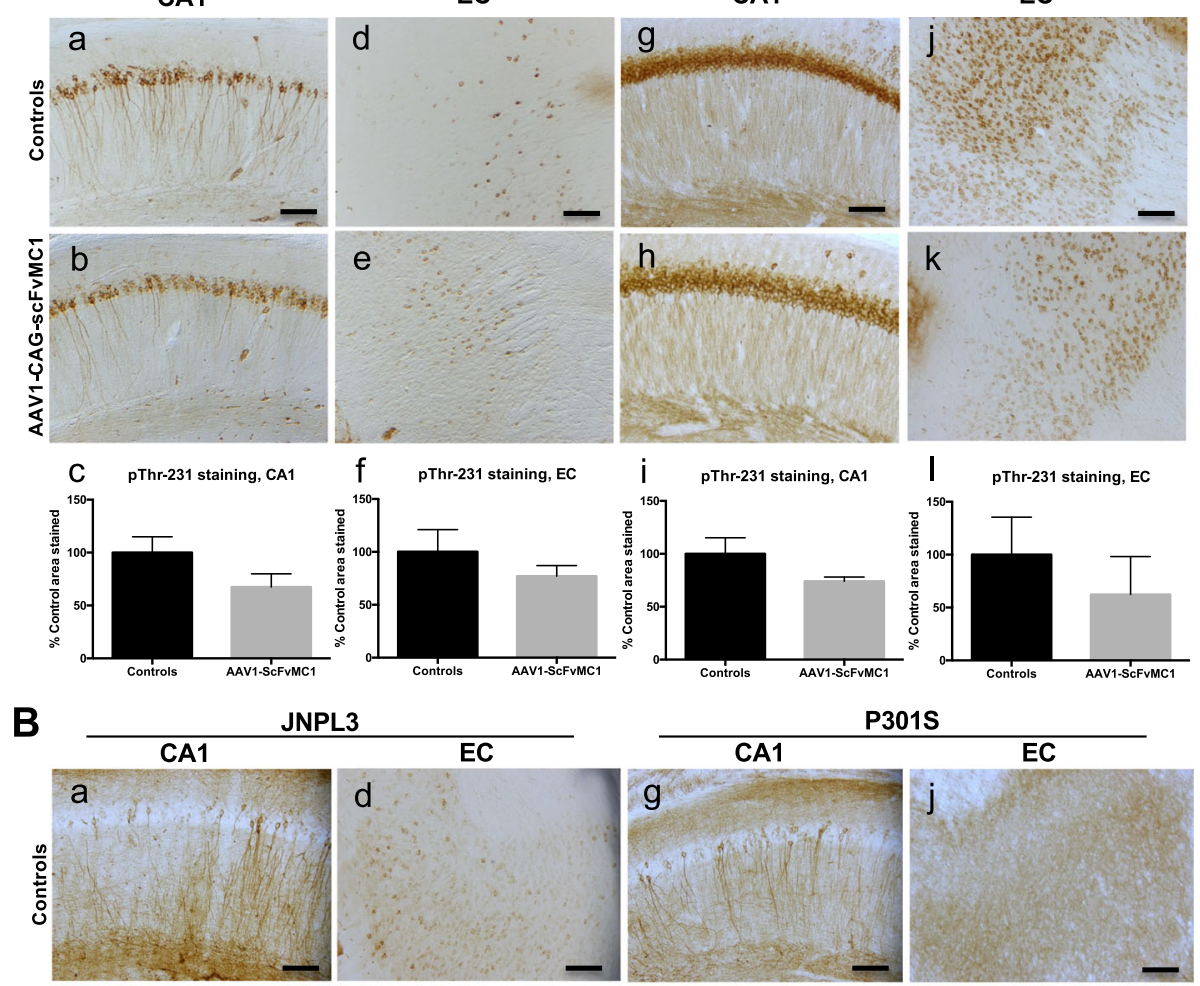

JNPL3
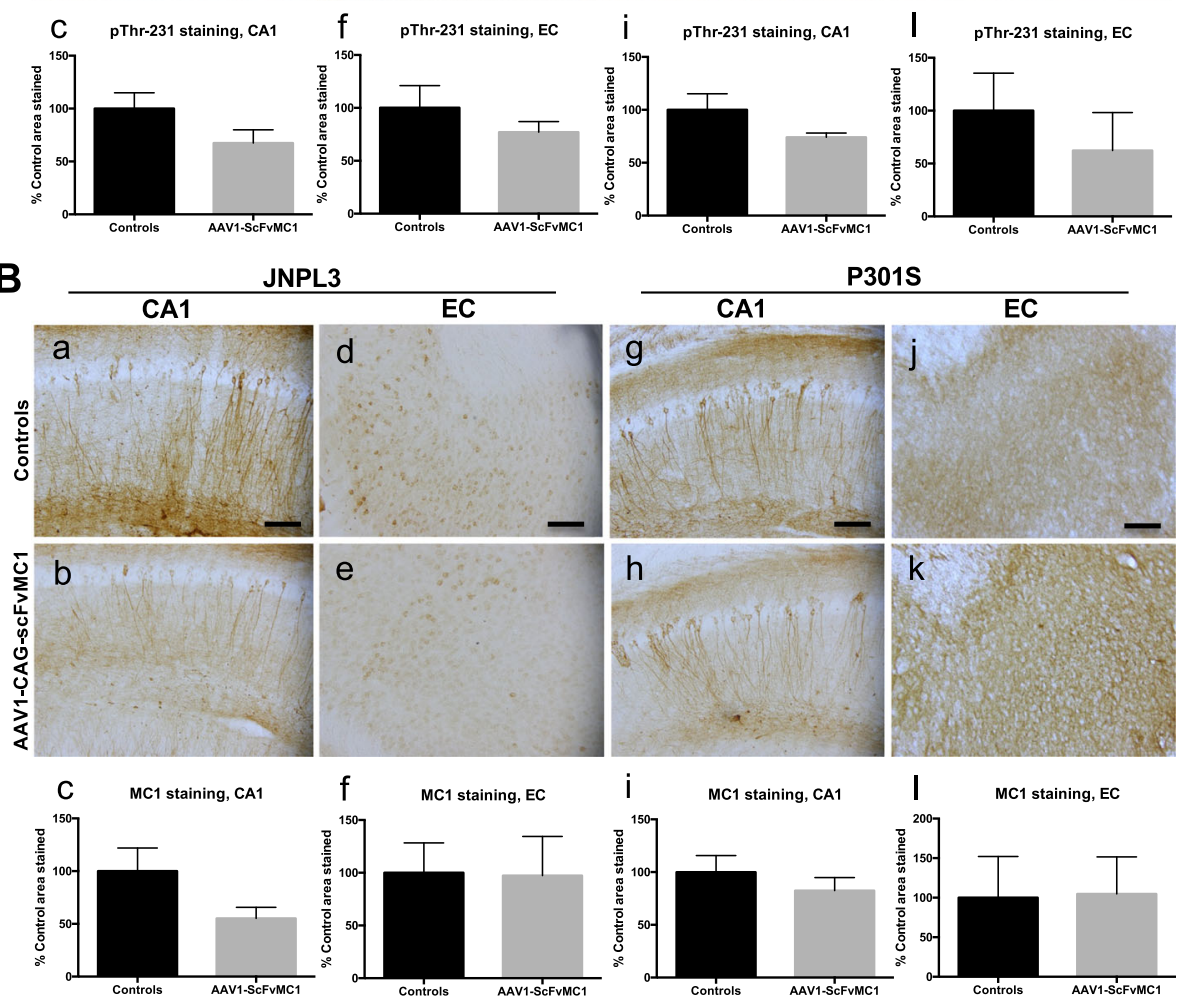

Fig. 6 Phospho-Threonine-231 and MC1-tau immunoreactivity in brain. Brains from P301S and JNPL3 animals were harvested and stained with either anti tau phospho-Thr231 antibody (A) or anti-MC1-tau (B). (A): a, b, d, e Representative images of CA1 hippocampal cell layer and entorhinal cortex (EC) stained with anti-pThr231. Control mice received AAV1-CAG-eGFP injection $(n=13)$. Treated mice were injected with AAV1-CAG-sCFvMC1 $(n=13)$. (A): $\mathbf{c , f}$ Semi-quantification of percentage of area stained by RZ3 shows a trend of reduction in the AAV1-CAG-scFvMC1 injected group, in the CA1 region of hippocampus in the JNPL3 (c, $p=0.1128$, unpaired $t$ test with Welch's correction); no trend to reduction is detected in the entorhinal cortex (EC) (f, $p=0.3458$, unpaired t test with Welch's correction). (A): $\mathbf{g}, \mathbf{h}, \mathbf{j}, \mathbf{k}$ Representative images of the CA1 region and entorhinal cortex (EC) from P301S; pThr-231 staining was performed as above. Control mice received AAV1-CAG-eGFP injection $(n=6)$, treated mice were injected with AAV1CAG-scFvMC1 $(n=6)$; $(\mathbf{A}) \mathbf{i}$, I Semi-quantification of percentage of area stained by RZ3 in P301S mice (i, $p=0.2403 ; \mathbf{I}, p=0.2251$; non parametric MannWhitney test). (B): $\mathbf{a}, \mathbf{b}, \mathbf{d}$, e Representative images of CA1 hippocampal cell layer and entorhinal cortex (EC) stained with anti-MC1 antibody. Control mice received AAV1-CAG-eGFP injection $(n=13)$. Treated mice were injected with AAV1-CAG-scFvMC1 $(n=13)(\mathbf{c}, \mathbf{f})$ Semi-quantification of percentage of area stained by MC1 shows a trend of reduction in the AAV1-CAG-sCFVMC1 injected group, in the CA1 region of hippocampus (c, $p=0.0996$; unpaired $t$ test with Welch's correction); no reduction is detected in the entorhinal cortex (EC) (f, $p=0.9558$; unpaired t test with Welch's correction) in JNPL3. (g, h, j, k) Representative images of the CA1 region and the entorhinal cortex (EC) from P301S; MC1 staining was performed as above. Control mice received AAV1-CAG-eGFP injection $(n=6)$, treated mice were injected with AAV1-CAG-sCFVMC1 $(n=6)$. (i, I) Semi-quantification of percentage of area stained by MC1 in P301S mice (i, $p=0.4127 ; \mathbf{I}, p=0.8413$; non parametric Mann-Whitney). (Olympus BH-2 bright field microscope; scale bar: $100 \mu \mathrm{m})$. Graphs are expressed as \% Control area stained, and means +/- SEM 


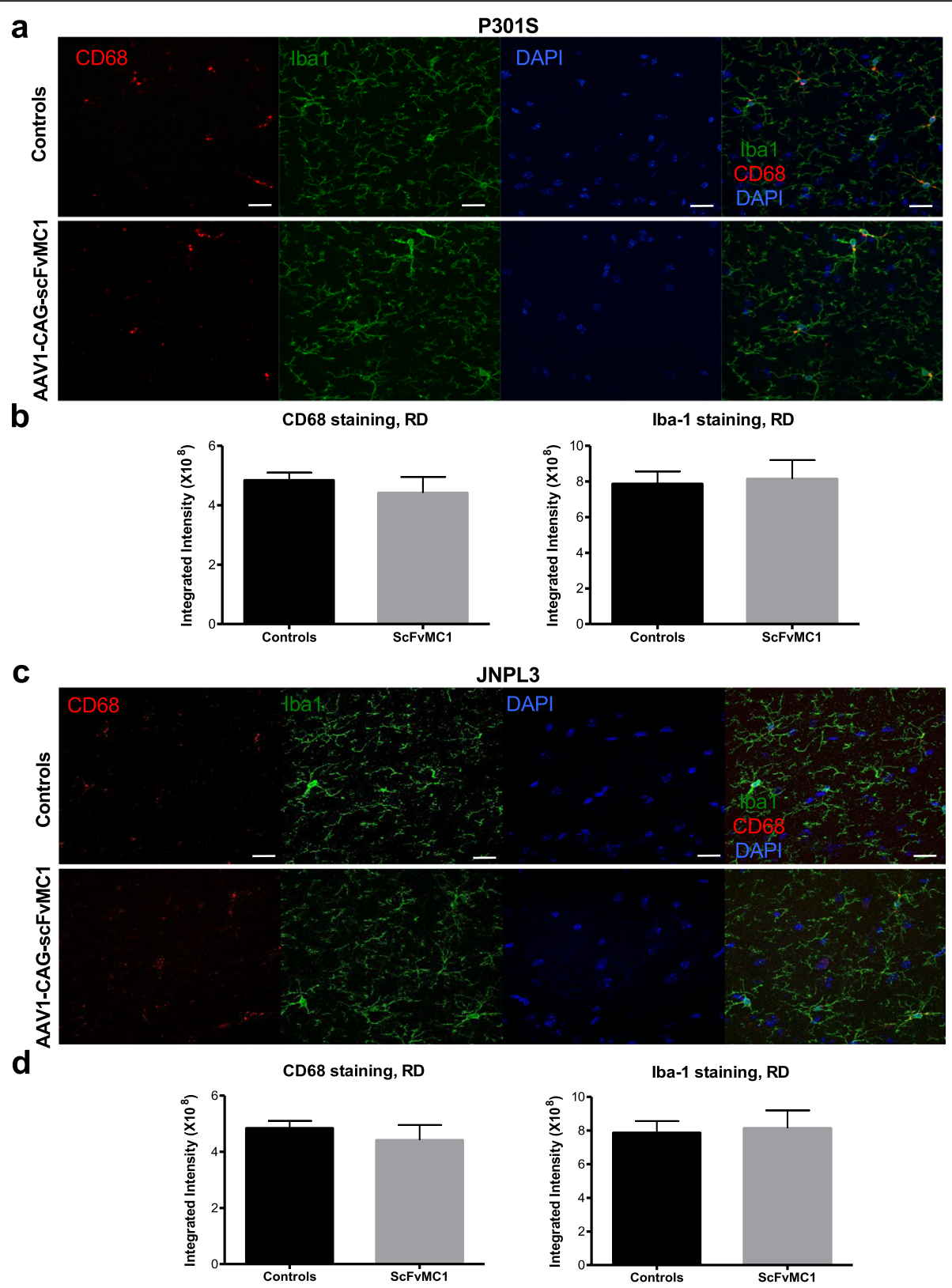

Fig. 7 Microglia activation state does not change upon scFvMC1 treatment. For analysis of activation state, sections were immunolabeled for CD68 and Iba-1 and imaged. a Representative image of microglia from P301S controls ( $n=6)$ (upper images) and AAV-sCFV-MC1 treated mice $(n=6)$ (lower images), in the stratum radiatum: CD68 lysosomal protein (red puncta), Iba1 (green), nuclei stained with DAPI (blue). b Quantification of Iba-1 and CD68 showed no significant differences between controls and treated mice ( $p=0.0931$ and $p=0.7771$ respectively, non parametric Mann-Whitney test). c Representative image of microglia from JNPL3 controls ( $n=13)$ (upper images) and AAV-scFV-MC1 treated mice $(n=13)$ (lower images), in the stratum radiatum. $\mathbf{d}$ Quantification of Iba-1 and CD68 showed no significant differences between controls and treated mice in JNPL3 mice ( $p=0.8857$ and $p=0.4857$ respectively, non parametric Mann-Whitney test). All images were obtained using Zeiss 880 confocal laser microscope, scale bar: $20 \mu \mathrm{m}$. Graphs are expressed as integrated density, and means +/- SEM

Supplementary Fig. 3c, d tau levels did not change upon treatment in both mice models.

\section{Discussion}

The use of antibody fragments has emerged as a promising approach to target both $A \beta$ and tau pathology in
Alzheimer's disease [32-34, 39, 40, 42-45, 85]. We have previously reported that intracranial administration of the vectorized anti-tau scFvMC1 was able to reduce different tau species in the JNPL3 transgenic animal model [43]. This study has set the basis for the development of a novel therapeutic approach aimed at advancing 


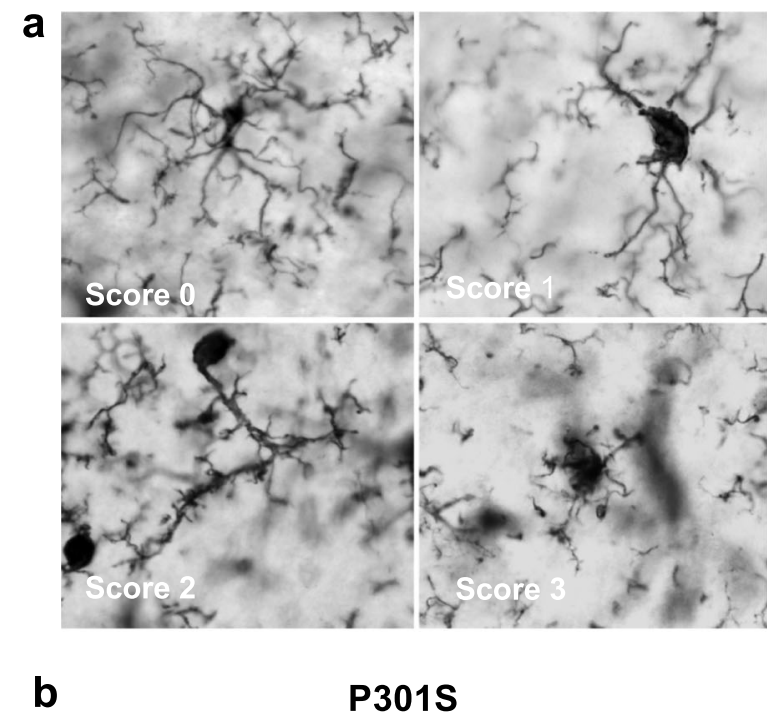

b

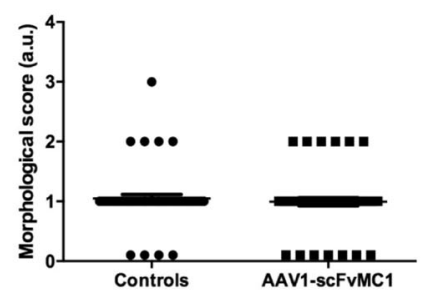

C

JNPL3

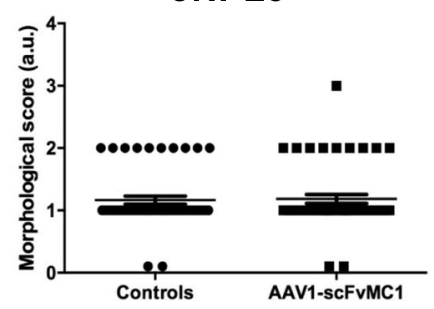

Fig. 8 Microglia morphology is unchanged upon treatment. Microglia processes morphology was assessed in both P301S and JNPL3 animals. a Representative images of Iba-1 positive microglia (stratum radiatum of the CA1 subfield of the hippocampus): the processes morphology was scored as 0 (> 15 thin processes with multiple branches), 1 (5-15 thick processes with branches), 2 (1-5 thick processes with few branches), 3 (no clear processes). $\mathbf{b}$ Microglia from P301S mice did not show any significant morphological changes comparing controls to treated mice. Each single point represents a single cell $(n=50$ cells per group, $p=$ 0.5671, non parametric Mann Whitney test). c No significant changes in microglia morphology were detected in the JNPL3 cohort $(n=50$ cells per group, $p=0.9628$, non parametric Mann Whitney test). Graphs are expressed as arbitrary unit (a.u.) and means +/- SEM. (Axiolmager Z1 microscope, Zeiss; 63x oil and $0.58 \mu \mathrm{m}$ z-steps)

peripheral administration of vectorized anti-tau scFv: in the present work, we show that IM injection of anti-tau $\mathrm{scFv}$ antibodies has potential for the treatment of tauopathies.

Few studies $[35,45]$ have previously demonstrated that intramuscular delivery of an anti- $\mathrm{A} \beta \mathrm{scFv}$ gene in an $\mathrm{AD}$ mouse model reduced amyloid deposits and ameliorated its learning and memory deficits without inducing discernible inflammation. In line with the current strategies, we have utilized AAV1 to express scFvMC1 in the striated muscle, to generate a peripheral niche and achieve long term production of the fragment antibody. We have extended our investigation to two different models of tauopathy, reaching significant reduction of insoluble and soluble tau species, total and phosphorylated, with regional and mouse strain differences. The following considerations are in order.

Detecting scFvMC1 in the brain parenchyma using antibodies against the Myc or His tags (see construct design in [43]) has proven challenging when the $\mathrm{scFv}$ is produced in the periphery $[35,45]$, as in the current work. Contrarily, in our previous publication, where the $\mathrm{scFv}$ was expressed directly in the brain, we were able to detect and track the diffusion of the scFv by both IHC and western blot in the 3 brain regions analyzed (hippocampus, cortex, hindbrain) [43]. We thus hypothesized that the local brain concentration of the peripherally generated $\mathrm{scFv}$ would not be enough for detection. Using this systemic approach we also failed to detect $\mathrm{scFv}$ in serum: it is plausible that, together with the detection limits of the assay employed, a certain amount of recombinant antibody might get misfolded and degraded, explaining why we can't visualize scFv in serum. When we conjugated the scFvMC1 to an IR dye and injected $100 \mu \mathrm{g}$ of it by IV route (less than half the dose normally injected in anti-tau passive immunotherapy experiments in mice, i.e. $10 \mathrm{mg} / \mathrm{kg}$ ), we were able to detect the compound in the brain, and similarly for the native MC1 antibody. Importantly, the ratio between the blood and brain parenchyma concentrations are in line with what seen by others [72, 74, 86, 87] for both the native and $\mathrm{scFv}$ antibody. Hence, these data suggest that our $\mathrm{scFv}$ is able to cross the $\mathrm{BBB}$ and reach its target, justifying its use in a systemic delivery paradigm. Consistent with this proof-of-concept experiment, the efficacy data show that peripherally generated scFvMC1 can reach the brain parenchyma to modulate tau levels, with no peripheral leakage of the transgene expression, conferring to this approach a relevant translational advantage.

Previous studies from our laboratory and others [14, 16] have investigated the effect of tau passive immunotherapy using peripherally injected native $\mathrm{MC1}$, in both JNPL3 and P301S mice. Comparing the present study to the previous ones is not trivial, given the different nature of the antibodies (full antibody vs scFv), the variability of the mice strains and the delivery routes employed. One important finding in the present work is the highly significant decrease of insoluble total and phosphorylated tau in cortex, with similar response in P301S and JNPL3 mice. P301S soluble tau was also reduced across the 

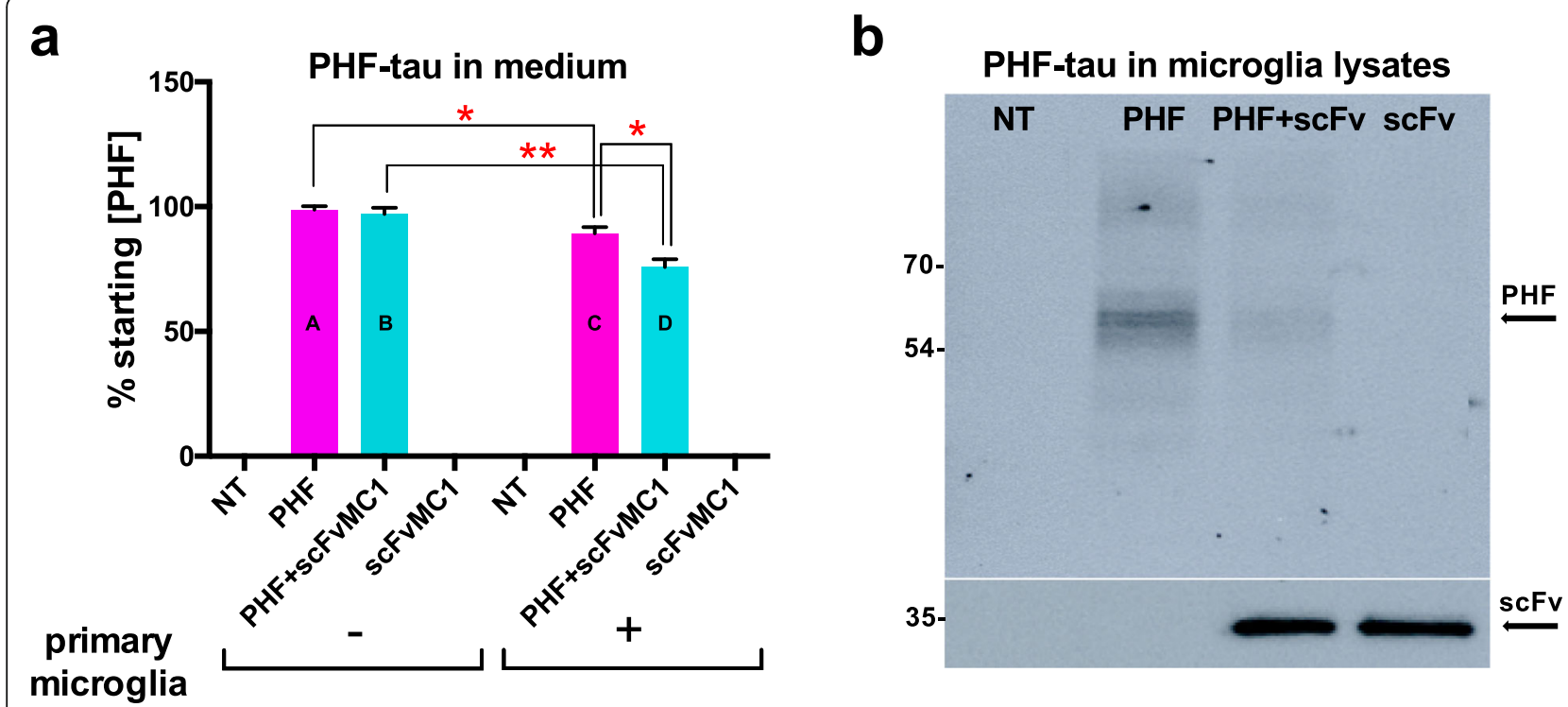

Fig. 9 Microglia uptake phosphorylated tau in vitro, facilitated by scFvMC1. (a) Primary mouse microglia (P2 C57BI/6 J pups) were treated in vitro for $2 \mathrm{~h}$ with PHF-tau +/- scFVMC1 (scFV/PHF ratio of 10/1). Total tau ELISA. Column A: PHF levels are expressed as \% of starting PHF

concentration measured after incubation on cell-free plates (- primary microglia); column B: amount of PHF in medium upon combination with scFvMC1, on cell-free plates (- primary microglia); column C and column D: PHF levels on microglia seeded plates (+ primary microglia), with or without scFvMC1. A vs $C\left({ }^{*} p<0.05\right.$, unpaired $t$ test with Welch's correction); C vs D ( ${ }^{*} p=0.0137$, unpaired t test with Welch's correction); B vs D ${ }^{* *} p=0.0019$ unpaired $t$ test with Welch's correction). Data are collected from three different experiments, with treatments run in quadruplicates. Graphs are means +/- SEM. (b, upper panel) Representative immuno-blotting (PHF1 antibody: anti-pSer396/404) of PHF-tau in the corresponding microglia lysates; (b, lower panel) scFvMC1 expression verified using anti Myc-tag antibody. NT is non treated microglia; PHF is microglia treated with PHF-tau; PHF + scFv is microglia co-treated with PHF-tau and scFvMC1; scFv is microglia treated with scFvMC1

brain, while oligomeric/aggregated tau was reduced mostly in the hippocampus. On the contrary, we did not observe any reduction of soluble and oligomeric species in the JNPL3 mice, and detected instead an increase of 2 phospho-tau epitopes in the hindbrain: we have no clear explanation at this point for the increase of soluble pSer202 (early p-tau epitope in AD) and pSer396/404 (late p-tau epitope in AD) tau in the JNPL3 HB, which is opposite to the other findings. Semi-quantitative analysis of RZ3 and MC1 immunohistochemistry staining on brain slices did not confirm the reduction of soluble tau detected biochemically by ELISA, in the P301S model, which is more sensitive to changes in soluble and insoluble tau. Taken together, these data point to regional and qualitative (soluble vs insoluble) differences in tau clearance in both mice models.

Indeed, mice strains may vary in the quantitative and topographic expression of the tau transgene (by virtue of promoters and copy numbers), rate of deposition of tau pathology and tau phosphorylation, inflammation/reactive changes and BBB permeability to the scFv. As previously reported, in contrast to the JNPL3 model, the P301S mice accumulate detectable levels of hyperphosphorylated tau species $(64-\mathrm{kDa})$ in soluble as well as insoluble fractions $[16,63]$. The precise reason for this difference between the pathological tau accumulation in the two models is unclear but may explain some discrepancy in the effect of anti-tau immunotherapy.

The use of antibodies to tackle tau pathology is fascinating, but may carry consequences in terms of eliciting an inflammatory response, by activation of immune cells via Fc receptor. In our system, the absence of the Fc region should prevent such occurrence. Indeed, we have not found any signs of microglia activation, nor we have found signs of peripheral tissue damage from the AAVscFvMC1 transduction, or increased pro-inflammatory cytokines levels in serum. Hence, so far, our strategy appears to be safe. However, the host immune system could pose some limitations, such as cellular immune responses to AAV vector and/or transgene. In this respect, the AAV field is moving into developing strategies to overcome immunogenicity produced by readministration [88]. In our study, we have detected antibodies directed to scFvMC1 in the JNPL3 cohort, which did not prevent efficacy, in line with what reported earlier [43].

Depending on the administration route, the dose, and/ or the AAV serotype, gene transfer can result in a deleterious immune response $[89,90]$ or tolerance $[61,91$, 92]. Two studies have shown that AAV1 IM injection can lead to regulatory $\mathrm{T}$ cells (Tregs) infiltration in injected muscles in AATD (Alpha-1 Antitrypsin 


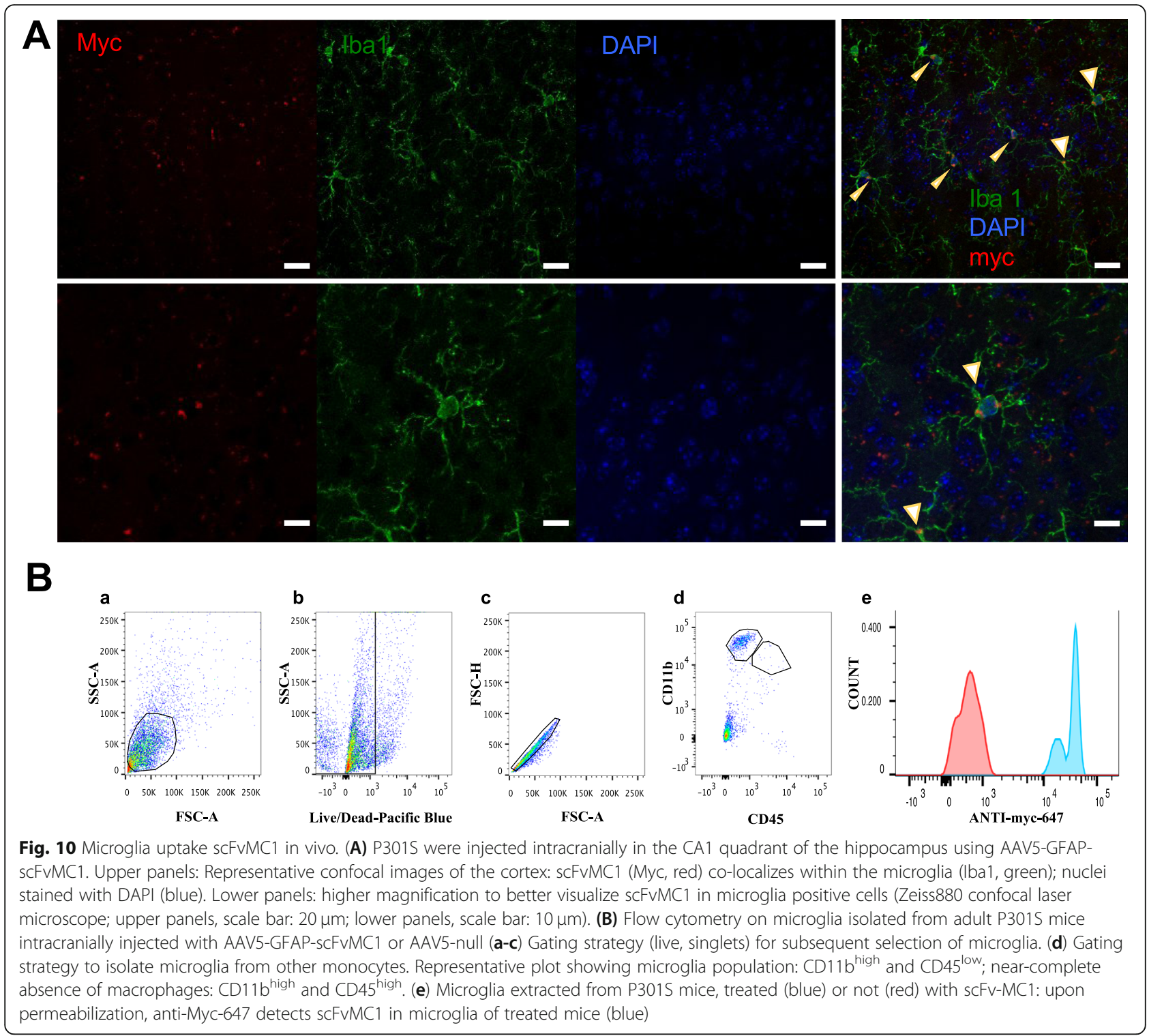

Deficiency) and LPLD (Lipoprotein Lipase Deficiency) patients [61, 91]. Gernoux et al. [92] have recently correlated results obtained in monkeys to those obtained with patients and further demonstrated that tolerance and persisting transgene expression after AAV1 gene transfer in muscle is mediated by Tregs and exhausted $\mathrm{T}$ cells. These findings support the IM approach proposed in the present study and its translational potential for humans.

One last mechanistic aspect was investigated: the role of microglia in clearing tau after scFvMC1 administration. Luo et al. [93] have reported that murine microglia rapidly internalize and degrade hyperphosphorylated pathological tau isolated from AD brain tissue in a timedependent manner in vitro, showing that the anti-tau monoclonal antibody $\mathrm{MC} 1$ had the ability to enhances microglia-mediated tau degradation in an Fc-dependent manner. Despite the lack of Fc in our scFvMC1, we wanted to investigate this mechanistic aspect, assuming that the microglia could still participate in the removal of the immunocomplex in our model. Thus, we took both an in vitro and in vivo approach to determine if microglia are able to uptake PHF (hyperphosphorylated aggregated tau) with or without the help of scFv. We found that in vitro, cultured microglia were able to uptake PHF-tau (confirming the previous study [93]) and scFvMC1 had the ability to enhances this process; furthermore, scFvMC1 accelerated the degradation of PHF tau in these cells. In vivo, we have been able to colocalize tau (anti-pThr231), scFvMC1 (anti-Myc) and microglia cells (anti-Iba1) after intracranial injection of $\mathrm{AAV}$-scFvMC1. It is plausible that multiple mechanisms contribute to the clearance of tau upon $\mathrm{scFv}$ 
immunotherapy and that microglia uptake is one of the routes the brain uses to remove pathological tau. Future experiments will investigate whether microglia or other CNS or peripheral cell types participate in the uptake of scFvMC1 and tau using receptors other than the FcR, i.e. toll-like receptors (TLRs), C-X3-C motif chemokine receptor 1 (CX3CR1), pattern-recognition receptors (PRRs), scavenger receptors, and complement protein $\mathrm{C} 1 \mathrm{q}$ receptor (C1q-R).

\section{Conclusions}

To our knowledge, this work provides the first description of vectorized anti-tau scFv exerting an effect in the brain upon intramuscular injection. More studies are planned to investigate whether the highly significant reduction of insoluble tau gained in both animal models will also ameliorate their behavioral phenotype. Also, from a clinical perspective, particularly attractive would be the ability to not only express the scFv but to switch it on or off at will, adding a layer of exogenous control to improve safety. Among the currently available inducer/repressor systems permitting control over gene expression, the tetracycline (Tet)-dependent system is by far the most advanced and most widely used, and has been already tested in association with AAV1 and locoregional muscle gene transfer in non-human primates showing no humoral or cellular responses against the transgene [94]: we will certainly explore this avenue in our system.

In addition, our work opens the field to future studies reaching beyond $\mathrm{scFv}$, to test engineered antibodies with multiple specificities and targets in the brain, using a simple and translatable approach.

Overall, given the limitations linked to conventional immunotherapy in neurodegenerative diseases, this work demonstrates the efficacy and the advantages of using intramuscular injection of vectorized $\mathrm{scFv}$ to target tau, and its relevant translational features, suggesting potential applications to other brain proteinopathies.

\section{Supplementary information}

Supplementary information accompanies this paper at https://doi.org/10. 1186/s40478-020-01003-7.

Additional file 1 Supplementary figure 1: Inflammatory status in peripheral organs. NF-kB immunoreactivity, marker of activated proinflammatory pathways, was evaluated on kidney, liver, tibialis muscle and heart: representative images of each organs do not show differences between controls and AAV1-SCFvMC1 treated mice (Controls = AAV1-CAGeGFP; Bright field microscope, scale bar: $300 \mu \mathrm{m}$ ).

Additional file $\mathbf{2}$ Supplementary figure 2. Phospho-tau and scFvMC1 co-localize in microglia. Representative confocal image of the stratum radiatum from P301S mice injected with AAV5-GFAP-scFvMC1. Astrocytes actively express SCFv-MC1 (Myc-red); lba1 positive microglia (green) shows co-localization of scFvMC1 (myc-red) and pTau (pThr231; blue): merge purple (white arrows). Zeiss 880 confocal laser microscope: merge image is $2 x$ crop of $40 \mathrm{X}$ magnification; scale bar: $20 \mu \mathrm{m}$.

Additional file $\mathbf{3}$ Supplementary figure $\mathbf{3}$ Anti-scFvMC1and tau in serum. $(\mathbf{a}, \mathbf{b})$ Antibodies anti-scFvMC1 were assessed using a specific immune sorbent assay: JNPL3 mice receiving the scFvMC1 exhibited a significant increase of anti-scFvMC1 in serum ${ }^{* * *} p=0.0005$, non parametric Mann-Whitney test) compared to the controls; no anti-scFvMC1 were detected in the P301S animals; $(\mathbf{c}, \mathbf{d})$ total tau levels were measured in serum at the end of the 4 month treatment: no significant changes were identified in both transgenic models, P301S and JNPL3. Graphs are expressed as Arbitrary Units $(\mathbf{a}, \mathbf{b})$ or \% Control (c, d), and means +/SEM.

\section{Abbreviations}

Ab: Antibody; AB: Amyloid-beta; AAV: Adeno-associated viral vector; AD: Alzheimer's disease; BBB: Blood-brain barrier; CNS: Central nervous system; Ctx: Cortex; EC: Entorhinal cortex; HB: Hind brain;

Hippo: Hippocampus; IM: Intramuscular; IV: Intravenous; LV: Lentiviral vector; mAbs: Monoclonal antibodies; PHF: Paired helical filaments; RD: Stratum radiatum; scFv: Single chain variable fragment; scFvMC1: Single chain variable fragment $M C 1$; Tregs: Regulatory $T$ cells; $V_{H}$ : Variable heavy chain; $V_{L}$ : Variable light chain

\section{Acknowledgements}

We thank Dr. Peter Davies for providing tau antibodies and PHF-tau. We would like to thank Dr. Czeslawa Kowal for helping with retro-orbital injections.

\section{Ethic approval and consent to participate}

All experiments were conducted under the institutional guidelines and were approved by the Institutional Animal Care and Use Committee at The Feinstein Institutes for Medical Research, Northwell Health.

\section{Authors' contributions}

FV performed experiments, analysed data and helped preparing the manuscript; JO performed experiments and helped preparing figures; BTV analysed and interpreted data; PM interpreted experiments and edited the manuscript; LG designed the overall project, analysed data, interpreted results and prepared the manuscript; $C D$ designed the overall project, performed experiments, analysed data, interpreted results and prepared the manuscript. The authors read and approved the final manuscript.

\section{Funding}

This study was supported by the National Institute of Health $(\mathrm{NIH})$ grant 1R56AG055479-01 to C. d'Abramo.

\section{Availability of data and materials}

The datasets used and/or analyzed during the current study are available from the corresponding authors upon reasonable request.

\section{Consent for publication}

Not applicable.

\section{Competing interests}

The authors declare no conflict of interest.

\section{Author details}

${ }^{1}$ Institute of Molecular Medicine, The Litwin-Zucker Center for Alzheimer's Disease \& Memory Disorder, The Feintein Institutes for Medical Research, Manhasset, NY, USA. ${ }^{2}$ Donald and Barbara Zucker School of Medicine at Hofstra/Northwell, Hempstead, NY, USA. ${ }^{3}$ Institute of Molecular Medicine, Center for Autoimmune and Musculoskeletal Disease, The Feinstein Institutes for Medical Research, Manhasset, USA. ${ }^{4}$ Northwell Health Neuroscience Institute, Northwell Health System, Manhasset, NY, USA.

Received: 15 May 2020 Accepted: 27 July 2020

Published online: 06 August 2020

References

1. Wang Y, Mandelkow E (2016) Tau in physiology and pathology. Nat Rev Neurosci 17(1):5-21 
2. Cleveland DW, Hwo SY, Kirschner MW (1977) Physical and chemical properties of purified tau factor and the role of tau in microtubule assembly. J Mol Biol 116(2):227-247

3. Buée $L$ et al (2000) Tau protein isoforms, phosphorylation and role in neurodegenerative disorders. Brain Res Brain Res Rev 33(1):95-130

4. Mandelkow EM, Mandelkow E (2012) Biochemistry and cell biology of tau protein in neurofibrillary degeneration. Cold Spring Harb Perspect Med 2(7): a006247

5. Rösler TW et al (2019) Four-repeat tauopathies. Prog Neurobiol 180:101644

6. Frost B, Jacks RL, Diamond MI (2009) Propagation of tau misfolding from the outside to the inside of a cell. J Biol Chem 284(19):12845-12852

7. Clavaguera F et al (2009) Transmission and spreading of tauopathy in transgenic mouse brain. Nat Cell Biol 11(7):909-913

8. Wu JW et al (2013) Small misfolded tau species are internalized via bulk endocytosis and anterogradely and retrogradely transported in neurons. J Biol Chem 288(3):1856-1870

9. Clavaguera F et al (2014) Peripheral administration of tau aggregates triggers intracerebral tauopathy in transgenic mice. Acta Neuropathol 127(2):299-301

10. Clavaguera F, Grueninger F, Tolnay M (2014) Intercellular transfer of tau aggregates and spreading of tau pathology: implications for therapeutic strategies. Neuropharmacology 76 Pt A:9-15

11. Clavaguera F et al (2013) Brain homogenates from human tauopathies induce tau inclusions in mouse brain. Proc Natl Acad Sci U S A 110(23): 9535-9540

12. de Calignon A et al (2012) Propagation of tau pathology in a model of early Alzheimer's disease. Neuron 73(4):685-697

13. Colin M et al (2020) From the prion-like propagation hypothesis to therapeutic strategies of anti-tau immunotherapy. Acta Neuropathol 139(1):3-25

14. d'Abramo C et al (2013) Tau passive immunotherapy in mutant P301L mice: antibody affinity versus specificity. PLoS One 8(4):e62402

15. d'Abramo C et al (2015) Passive immunization in JNPL3 transgenic mice using an Array of Phospho-tau specific antibodies. PLoS One 10(8):e0135774

16. Chai $X$ et al (2011) Passive immunization with anti-tau antibodies in two transgenic models: reduction of tau pathology and delay of disease progression. J Biol Chem 286(39):34457-34467

17. Boutajangout $\mathrm{A}$ et al (2011) Passive immunization targeting pathological phospho-tau protein in a mouse model reduces functional decline and clears tau aggregates from the brain. J Neurochem 118(4):658-667

18. Boutajangout A, Quartermain D, Sigurdsson EM (2010) Immunotherapy targeting pathological tau prevents cognitive decline in a new tangle mouse model. J Neurosci 30(49):16559-16566

19. Asuni AA et al (2007) Immunotherapy targeting pathological tau conformers in a tangle mouse model reduces brain pathology with associated functional improvements. J Neurosci 27(34):9115-9129

20. Sankaranarayanan S et al (2015) Passive immunization with phospho-tau antibodies reduces tau pathology and functional deficits in two distinct mouse tauopathy models. PLoS One 10(5):e0125614

21. Castillo-Carranza DL et al (2014) Passive immunization with tau oligomer monoclonal antibody reverses tauopathy phenotypes without affecting hyperphosphorylated neurofibrillary tangles. J Neurosci 34(12):4260-4272

22. Albert $\mathrm{M}$ et al (2019) Prevention of tau seeding and propagation by immunotherapy with a central tau epitope antibody. Brain 142(6):1736-1750

23. Yanamandra K et al (2015) Anti-tau antibody reduces insoluble tau and decreases brain atrophy. Ann Clin Transl Neurol 2(3):278-288

24. Yanamandra K et al (2013) Anti-tau antibodies that block tau aggregate seeding in vitro markedly decrease pathology and improve cognition in vivo. Neuron 80(2):402-414

25. Courade JP et al (2018) Epitope determines efficacy of therapeutic anti-tau antibodies in a functional assay with human Alzheimer tau. Acta Neuropathol 136(5):729-745

26. Dai CL et al (2018) Tau passive immunization blocks seeding and spread of Alzheimer hyperphosphorylated tau-induced pathology in $3 \times \mathrm{Tg}-\mathrm{AD}$ mice. Alzheimers Res Ther 10(1):13

27. Pardridge WM (2012) Drug transport across the blood-brain barrier. J Cereb Blood Flow Metab 32(11):1959-1972

28. Pardridge WM (2009) Alzheimer's disease drug development and the problem of the blood-brain barrier. Alzheimers Dement 5(5):427-432

29. Wilcock DM, Colton CA (2009) Immunotherapy, vascular pathology, and microhemorrhages in transgenic mice. CNS Neurol Disord Drug Targets 8(1): 50-64
30. Boche D et al (2008) Consequence of Abeta immunization on the vasculature of human Alzheimer's disease brain. Brain 131(Pt 12):3299-3310

31. Frenkel D, Katz O, Solomon B (2000) Immunization against Alzheimer's beta -amyloid plaques via EFRH phage administration. Proc Natl Acad Sci U S A 97(21):11455-11459

32. Liu $R$ et al (2004) Single chain variable fragments against beta-amyloid (Abeta) can inhibit Abeta aggregation and prevent abeta-induced neurotoxicity. Biochemistry 43(22):6959-6967

33. Fukuchi $\mathrm{K}$ et al (2006) Anti-Abeta single-chain antibody delivery via adenoassociated virus for treatment of Alzheimer's disease. Neurobiol Dis 23(3):502-511

34. Levites $Y$ et al (2006) Intracranial adeno-associated virus-mediated delivery of anti-pan amyloid beta, amyloid beta40, and amyloid beta42 single-chain variable fragments attenuates plaque pathology in amyloid precursor protein mice. J Neurosci 26(46):11923-11928

35. Wang YJ et al (2009) Intramuscular delivery of a single chain antibody gene reduces brain Abeta burden in a mouse model of Alzheimer's disease. Neurobiol Aging 30(3):364-376

36. Ryan DA et al (2010) Abeta-directed single-chain antibody delivery via a serotype-1 AAV vector improves learning behavior and pathology in Alzheimer's disease mice. Mol Ther 18(8):1471-1481

37. Wang XP et al (2009) Conformation-dependent single-chain variable fragment antibodies specifically recognize beta-amyloid oligomers. FEBS Lett 583(3):579-584

38. Boado RJ et al (2010) IgG-single chain Fv fusion protein therapeutic for Alzheimer's disease: expression in $\mathrm{CHO}$ cells and pharmacokinetics and brain delivery in the rhesus monkey. Biotechnol Bioeng 105(3):627-635

39. Fernandez-Funez $P$ et al (2015) Anti-A $\beta$ single-chain variable fragment antibodies exert synergistic neuroprotective activities in drosophila models of Alzheimer's disease. Hum Mol Genet 24(21):6093-6105

40. Liu W et al (2016) Vectored intracerebral immunization with the anti-tau monoclonal antibody PHF1 markedly reduces tau pathology in mutant tau transgenic mice. J Neurosci 36(49):12425-12435

41. Gallardo G et al (2019) Targeting tauopathy with engineered tau-degrading intrabodies. Mol Neurodegener 14(1):38

42. Ising C et al (2017) Correction: AAV-mediated expression of anti-tau scFvs decreases tau accumulation in a mouse model of tauopathy. J Exp Med 214(7):2163

43. Vitale F et al (2018) Anti-tau conformational scFv MC1 antibody efficiently reduces pathological tau species in adult JNPL3 mice. Acta Neuropathol Commun 6(1):82

44. Spencer B et al (2018) Selective targeting of 3 repeat tau with brain penetrating single chain antibodies for the treatment of neurodegenerative disorders. Acta Neuropathol 136(1):69-87

45. Yang J et al (2013) Muscle-directed anti-Aß single-chain antibody delivery via $A A V 1$ reduces cerebral $A B$ load in an Alzheimer's disease mouse model. J Mol Neurosci 49(2):277-288

46. Bird RE et al (1988) Single-chain antigen-binding proteins. Science 242(4877):423-426

47. Huston JS et al (1993) Medical applications of single-chain antibodies. Int Rev Immunol 10(2-3):195-217

48. Holliger P. Hudson PJ (2005) Engineered antibody fragments and the rise of single domains. Nat Biotechnol 23(9):1126-1136

49. Wörn A, Plückthun A (2001) Stability engineering of antibody single-chain Fv fragments. J Mol Biol 305(5):989-1010

50. Arndt KM, Müller KM, Plückthun A (1998) Factors influencing the dimer to monomer transition of an antibody single-chain Fv fragment. Biochemistry 37(37):12918-12926

51. Milone MC, O'Doherty U (2018) Clinical use of lentiviral vectors. Leukemia 32(7):1529-1541

52. Rafii MS et al (2018) Adeno-associated viral vector (serotype 2)-nerve growth factor for patients with Alzheimer disease: a randomized clinical trial. JAMA Neurol 75(7):834-841

53. Herzog CD et al (2011) Gene transfer provides a practical means for safe, long-term, targeted delivery of biologically active neurotrophic factor proteins for neurodegenerative diseases. Drug Deliv Transl Res 1(5):361-382

54. LeWitt PA et al (2011) AAV2-GAD gene therapy for advanced Parkinson's disease: a double-blind, sham-surgery controlled, randomised trial. Lancet Neurol 10(4):309-319

55. Hitti FL et al (2019) Human gene therapy approaches for the treatment of Parkinson's disease: an overview of current and completed clinical trials. Parkinsonism Relat Disord 66:16-24 
56. McPhee SW et al (2006) Immune responses to AAV in a phase I study for Canavan disease. J Gene Med 8(5):577-588

57. Christine CW et al (2009) Safety and tolerability of putaminal AADC gene therapy for Parkinson disease. Neurology 73(20):1662-1669

58. Mendell JR et al (2017) Single-dose gene-replacement therapy for spinal muscular atrophy. N Engl J Med 377(18):1713-1722

59. Rivière C, Danos O, Douar AM (2006) Long-term expression and repeated administration of AAV type 1,2 and 5 vectors in skeletal muscle of immunocompetent adult mice. Gene Ther 13(17):1300-1308

60. Mueller $C$ et al (2017) 5 year expression and neutrophil defect repair after gene therapy in Alpha-1 antitrypsin deficiency. Mol Ther 25(6):1387-1394

61. Mueller C et al (2013) Human Treg responses allow sustained recombinant adeno-associated virus-mediated transgene expression. J Clin Invest 123(12): 5310-5318

62. Lewis J et al (2000) Neurofibrillary tangles, amyotrophy and progressive motor disturbance in mice expressing mutant (P301L) tau protein. Nat Genet 25(4):402-405

63. Allen B et al (2002) Abundant tau filaments and nonapoptotic neurodegeneration in transgenic mice expressing human P301S tau protein. J Neurosci 22(21):9340-9351

64. Acker CM et al (2013) Sensitive quantitative assays for tau and phospho-tau in transgenic mouse models. Neurobiol Aging 34(1):338-350

65. Forest SK et al (2013) Methods for measuring tau pathology in transgenic mouse models. J Alzheimers Dis 33(2):463-471

66. Schafer DP et al (2012) Microglia sculpt postnatal neural circuits in an activity and complement-dependent manner. Neuron 74(4):691-705

67. Nestor J et al (2018) Lupus antibodies induce behavioral changes mediated by microglia and blocked by ACE inhibitors. J Exp Med 215(10):2554-2566

68. Chan $\mathrm{K}$ et al (2020) Lupus autoantibodies act as positive allosteric modulators at GluN2A-containing NMDA receptors and impair spatial memory. Nat Commun 11(1):1403

69. Greenberg SG, Davies P (1990) A preparation of Alzheimer paired helical filaments that displays distinct tau proteins by polyacrylamide gel electrophoresis. Proc Natl Acad Sci U S A 87(15):5827-5831

70. Bennett ML et al (2016) New tools for studying microglia in the mouse and human CNS. Proc Natl Acad Sci U S A 113(12):E1738-E1746

71. d'Abramo C et al (2016) Detecting tau in serum of transgenic animal models after tau immunotherapy treatment. Neurobiol Aging 37:58-65

72. Paul SM (2011) Therapeutic antibodies for brain disorders. Sci Transl Med 3(84):84ps20

73. Esquerda-Canals G, Martí-Clúa J, Villegas S (2019) Pharmacokinetic parameters and mechanism of action of an efficient anti-A $\beta$ single chain antibody fragment. PLoS One 14(5):e0217793

74. Bard F et al (2000) Peripherally administered antibodies against amyloid beta-peptide enter the central nervous system and reduce pathology in a mouse model of Alzheimer disease. Nat Med 6(8):916-919

75. Thompson EJ, Keir G (1990) Laboratory investigation of cerebrospinal fluid proteins. Ann Clin Biochem 27(Pt 5):425-435

76. Haidet AM et al (2008) Long-term enhancement of skeletal muscle mass and strength by single gene administration of myostatin inhibitors. Proc Natl Acad Sci U S A 105(11):4318-4322

77. Hammond TR et al (2019) Single-cell RNA sequencing of microglia throughout the mouse lifespan and in the injured brain reveals complex cell-state changes. Immunity 50(1):253-271.e6

78. Hammond TR, Robinton D, Stevens B (2018) Microglia and the brain: complementary Partners in Development and Disease. Annu Rev Cell Dev Biol 34:523-544

79. Shi Y et al (2019) Microglia drive APOE-dependent neurodegeneration in a tauopathy mouse model. J Exp Med 216(11):2546-2561

80. Bennett RE et al (2018) Partial reduction of microglia does not affect tau pathology in aged mice. J Neuroinflammation 15(1):311

81. Jicha GA et al (1997) Alz-50 and MC-1, a new monoclonal antibody raised to paired helical filaments, recognize conformational epitopes on recombinant tau. J Neurosci Res 48(2):128-132

82. Bohlen CJ, Bennett FC, Bennett ML (2019) Isolation and culture of microglia. Curr Protoc Immunol 125(1):e70

83. Bohlen CJ et al (2017) Diverse requirements for microglial survival, specification, and function revealed by defined-medium cultures. Neuron 94(4):759-773.e8
84. DeMattos RB et al (2002) Brain to plasma amyloid-beta efflux: a measure of brain amyloid burden in a mouse model of Alzheimer's disease. Science 295(5563):2264-2267

85. Nisbet RM et al (2017) Combined effects of scanning ultrasound and a tauspecific single chain antibody in a tau transgenic mouse model. Brain 140(5):1220-1230

86. Freund J (1930) Accumulation of antibodies in the central nervous system. J Exp Med 51(6):889-902

87. Atwal JK et al (2011) A therapeutic antibody targeting BACE1 inhibits amyloid- $\beta$ production in vivo. Sci Transl Med 3(84):84ra43

88. Meliani A et al (2018) Antigen-selective modulation of AAV immunogenicity with tolerogenic rapamycin nanoparticles enables successful vector readministration. Nat Commun 9(1):4098

89. Mingozzi F et al (2007) CD8(+) T-cell responses to adeno-associated virus capsid in humans. Nat Med 13(4):419-422

90. Hui DJ et al (2015) AAV capsid CD8+ T-cell epitopes are highly conserved across AAV serotypes. Mol Ther Methods Clin Dev 2:15029

91. Ferreira $V$ et al (2014) Immune responses to intramuscular administration of alipogene tiparvovec (AAV1-LPL(S447X)) in a phase II clinical trial of lipoprotein lipase deficiency gene therapy. Hum Gene Ther 25(3):180-188

92. Gernoux $\mathrm{G}$ et al (2020) Muscle-directed delivery of an AAV1 vector leads to capsid-specific T cell exhaustion in nonhuman primates and humans. Mol Ther 28(3):747-757

93. Luo W et al (2015) Microglial internalization and degradation of pathological tau is enhanced by an anti-tau monoclonal antibody. Sci Rep 5:11161

94. Guilbaud M et al (2019) Five years of successful inducible transgene expression following Locoregional adeno-associated virus delivery in nonhuman primates with no detectable immunity. Hum Gene Ther 30(7): $802-813$

\section{Publisher's Note}

Springer Nature remains neutral with regard to jurisdictional claims in published maps and institutional affiliations.

Ready to submit your research? Choose BMC and benefit from:

- fast, convenient online submission

- thorough peer review by experienced researchers in your field

- rapid publication on acceptance

- support for research data, including large and complex data types

- gold Open Access which fosters wider collaboration and increased citations

- maximum visibility for your research: over $100 \mathrm{M}$ website views per year

At BMC, research is always in progress.

Learn more biomedcentral.com/submissions 